TRANSACTIONS OF THE

AMERICAN MATHEMATICAL SOCIETY

Volume 358, Number 9, September 2006, Pages 3751-3780

S 0002-9947(06)03933-X

Article electronically published on April 11, 2006

\title{
DIMENSION OF HYPERBOLIC MEASURES OF RANDOM DIFFEOMORPHISMS
}

\author{
PEI-DONG LIU AND JIAN-SHENG XIE
}

\begin{abstract}
We consider dynamics of compositions of stationary random $C^{2}$ diffeomorphisms. We will prove that the sample measures of an ergodic hyperbolic invariant measure of the system are exact dimensional. This is an extension to random diffeomorphisms of the main result of Barreira, Pesin and Schmeling (1999), which proves the Eckmann-Ruelle dimension conjecture for a deterministic diffeomorphism.
\end{abstract}

\section{INTRODUCTION}

Let $M$ be a smooth compact Riemannian manifold without boundary. Let $f$ be a $C^{1+\alpha}$ diffeomorphism on $M$, and $\mu$ an ergodic $f$-invariant measure. An important open problem in dimension theory of dynamical systems for about 15 years is the existence for $\mu$-a.e. $x$ of the limit

$$
d_{\mu}(x):=\lim _{\rho \rightarrow 0} \frac{\log \mu(B(x, \rho))}{\log \rho}
$$

when $\mu$ is hyperbolic, i.e., all the Lyapunov exponents of $f$ are nonzero at $\mu$ almost every point. This is often referred to as the Eckmann-Ruelle conjecture, and an affirmative solution has been given in Barreira, Pesin and Schmeling [3], by exploiting previous substantial breakthroughs, especially Ledrappier and Young 8], and also by establishing a new and nontrivial property, called asymptotically "almost" local product structure, of the hyperbolic ergodic measure $\mu . d_{\mu}(x)$ is called the pointwise dimension of $\mu$ at $x$ when the limit exists. We refer the reader to 3 ] for a nice overview of the subject.

Our purpose in this paper is to extend the main result of [3] to the setting of random dynamical systems (RDS) generated by compositions of random diffeomorphisms (see Kifer [7, Arnold [1] or the survey Liu 9] for the background; an RDS of this kind reduces to a deterministic diffeomorphism when the distribution of the random diffeomorphisms is Dirac, i.e., is supported by a single point). In view of the importance of the Eckmann-Ruelle conjecture, the authors of the present paper think that such an extension deserves to be explicitly exposed, though the proof uses essentially the same strategy as that of [3]. We remark that, for accessibility

Received by the editors January 16, 2004 and, in revised form, April 11, 2004.

2000 Mathematics Subject Classification. Primary 37C45; Secondary 37H15.

Key words and phrases. Random dynamical systems, Eckmann-Ruelle conjecture, hyperbolic measure.

This work was supported by the 973 Fund of China for Nonlinear Science and the NSFDYS. 
of the proofs both of the deterministic and the random case, considerable further treatments are presented in this article.

This paper is organized in the following way. In Section 2 we introduce the setup of compositions of stationary random diffeomorphisms, present related notions and some preliminary results. We also give an extension to this setup of the generalized entropy formula of Ledrappier and Young 8. A detailed proof of such an extension was given in 13 for the more restricted i.i.d. (independent and identically distributed) random diffeomorphisms. The proof for the present setup is almost the same; we will indicate the modifications needed and omit the proof. The main result is stated in Section 3 and its proof is given in Sections 4-8.

\section{Notions AND PRELIMINARY RESULTS}

In this paper $M$ will always be a smooth and compact Riemannian manifold without boundary. Let $(\Omega, \mathcal{F}, P)$ be a probability space and $\theta:(\Omega, \mathcal{F}, P) \hookleftarrow$ an invertible measure-preserving transformation. For the need to work with Lebesgue spaces, we will assume further that $(\Omega, \mathcal{F}, P, \theta)$ is a Polish system, by which we mean that $\Omega$ is a Polish space (i.e., a separable topological space with a complete metric) and $\mathcal{F}$ is its Borel $\sigma$-algebra. Let $\operatorname{Diff}^{2}(M)$ be the space of $C^{2}$ diffeomorphisms of $M$, endowed with the usual $C^{2}$ topology and the Borel $\sigma$-algebra. Let

$$
F:(\Omega, \mathcal{F}, P) \rightarrow \operatorname{Diff}^{2}(M), \quad \omega \mapsto F(\omega)=: f_{\omega}
$$

be a measurable map and define

$$
f_{\omega}^{n}= \begin{cases}f_{\theta^{n-1} \omega} \circ \cdots \circ f_{\theta \omega} \circ f_{\omega} & \text { if } n>0, \\ \mathrm{id} & \text { if } n=0, \\ \left(f_{\theta^{n} \omega}\right)^{-1} \circ \cdots \circ\left(f_{\theta^{-2} \omega}\right)^{-1} \circ\left(f_{\theta^{-1} \omega}\right)^{-1} & \text { if } n<0 .\end{cases}
$$

We are concerned with the dynamical behavior of $f_{\omega}^{n}, n \in \mathbf{Z}$, for $P$-a.e. $\omega$, and this setup will be referred to as an $R D S$, still denoted by $F$, on $M$ over $(\Omega, \mathcal{F}, P, \theta)$.

Let $F$ be as given above. Define

$$
\Theta: \Omega \times M \rightarrow \Omega \times M, \quad(\omega, x) \mapsto\left(\theta \omega, f_{\omega} x\right)
$$

and call it the skew product transformation associated with $F$. Denote by $\mathcal{B}$ the Borel $\sigma$-algebra of $M$. An invariant measure of $F$ is defined to be a probability measure $\mu$ on $(\Omega \times M, \mathcal{F} \times \mathcal{B})$ which has marginal $P$ on $\Omega$ and is invariant under $\Theta$, and furthermore, $\mu$ is said to be ergodic if $\Theta:(\Omega \times M, \mu) \hookleftarrow$ is ergodic.

In the sequel $\mu$ is always an $F$-invariant measure (such measures always exist; see [1]). Since $\Omega$ is a Polish space, $(\Omega \times M, \mu)$ with the $\mu$-completion of $\mathcal{F} \times \mathcal{B}$ constitutes a Lebesgue space. According to Rokhlin [14, one can speak of the conditional measure $\mu_{\omega}$ of $\mu$ on $\{\omega\} \times M$ (identified with $M$ ) for $P$-a.e. $\omega$. $\mu_{\omega}$, $\omega \in \Omega$ are $P$-mod 0 uniquely defined and are called the sample measures of $\mu$. They clearly have the invariance property

$$
f_{\omega} \mu_{\omega}=\mu_{\theta \omega}, \quad P \text {-a.e. } \omega,
$$

and they could be regarded as natural "invariant" measures associated with individual realizations of the system $(F, \mu)$.

For every finite measurable partition $\eta$ of $M$, the limit

$$
h_{\mu}(F, \eta) \stackrel{\text { def }}{=} \lim _{n \rightarrow \infty} \frac{1}{n} \int H_{\mu_{\omega}}\left(\bigvee_{k=0}^{n-1}\left(f_{\omega}^{k}\right)^{-1} \eta\right) d P(\omega)
$$


always exists (see [4), and the number

$$
h_{\mu}(F):=\sup \left\{h_{\mu}(F, \eta): \eta \text { is a finite partition of } M\right\}
$$

is called the (measure-theoretic) entropy of $(F, \mu)$. It turns out that the entropy $h_{\mu}(F)$ coincides with the conditional entropy of $\Theta$ with respect to $\pi^{-1} \mathcal{F}$, i.e.,

$$
h_{\mu}(F)=h_{\mu}^{\pi^{-1} \mathcal{F}}(\Theta),
$$

where $\pi: \Omega \times M \rightarrow \Omega$ is the projection on the first factor (see [7, Theorem II.1.4] or [4, Theorem 3.1]).

Throughout this paper, it is always assumed that the RDS $F$ is given such that

$$
\int_{\Omega}\left(\log ^{+}\left|f_{\omega}\right|_{C^{2}}+\log ^{+}\left|\left(f_{\omega}\right)^{-1}\right|_{C^{2}}\right) d P<+\infty
$$

where $|g|_{C^{2}}$ denotes the usual $C^{2}$ norm of $g \in C^{2}(M, M)$ (see, e.g., Liu and Qian 11] for the definition). The Oseledec multipicative ergodic theorem applied to $\Theta:(\Omega \times M, \mu) \hookleftarrow$ tells us that for $\mu$-a.e. $(\omega, x)$ there exist numbers

$$
+\infty>\lambda_{1}(\omega, x)>\lambda_{2}(\omega, x)>\cdots>\lambda_{r(\omega, x)}(\omega, x)>-\infty
$$

and a splitting of $T_{x} M$

$$
T_{x} M=E_{1}(\omega, x) \oplus E_{2}(\omega, x) \oplus \cdots \oplus E_{r(\omega, x)}(\omega, x)
$$

(all measurable in $(\omega, x)$ ) such that

$$
\lim _{n \rightarrow \pm \infty} \frac{1}{n} \log \left|T_{x} f_{\omega}^{n} v\right|=\lambda_{i}(\omega, x)
$$

for all $v \in E_{i}(\omega, x) \backslash\{0\}, 1 \leq i \leq r(\omega, x)$. The numbers $\lambda_{i}(\omega, x), 1 \leq i \leq r(\omega, x)$, are called the Lyapunov exponents of $F$ at $(\omega, x)$, and $m_{i}(\omega, x):=\operatorname{dim} E_{i}(\omega, x)$ is called the multiplicity of $\lambda_{i}(\omega, x)$.

Let $u(\omega, x)$ be the number of positive Lyapunov exponents at $(\omega, x)$. Then for $\mu$-a.e. $(\omega, x)$, if $u(\omega, x)>0$, the set

$$
W^{u, i}(\omega, x):=\left\{y \in M: \varlimsup_{n \rightarrow+\infty} \frac{1}{n} \log d\left(f_{\omega}^{-n} x, f_{\omega}^{-n} y\right) \leq-\lambda_{i}(\omega, x)\right\}
$$

is a $C^{1,1}$ immersed $\sum_{k=1}^{i} m_{k}(\omega, x)$-dimensional submanifold of $M$ for each $1 \leq i \leq$ $u(\omega, x)$, and it is called the $i^{\text {th }}$-unstable manifold of $F$ at $(\omega, x) . W^{u, u(\omega, x)}(\omega, x)$ coincides with

$$
W^{u}(\omega, x):=\left\{y \in M: \varlimsup_{n \rightarrow+\infty} \frac{1}{n} \log d\left(f_{\omega}^{-n} x, f_{\omega}^{-n} y\right)<0\right\}
$$

and is called the unstable manifold of $F$ at $(\omega, x)$. Define $W^{u}(\omega, x)=\{x\}$ if $u(\omega, x)=0$.

Let $1 \leq i \leq \operatorname{dim} M$ and let $\eta^{i}$ be a measurable partition of the set $\Lambda_{i}:=\{(\omega, x) \in$ $\Omega \times M: W^{u, i}(\omega, x)$ is well defined $\}$, subordinate to the $W^{u, i}$-manifolds of $(F, \mu)$ (see [13] or [11] for the definition), and so that $\eta^{i} \geq\left.\{\{\omega\} \times M: \omega \in \Omega\}\right|_{\Lambda_{i}}$. Denote by $\mu_{(\omega, x)}^{i}$ the conditional measure of $\left.\mu\right|_{\Lambda_{i}}$ on $\eta^{i}(\omega, x)=:\{\omega\} \times \eta_{\omega}^{i}(x)$ (identified with $\left.\eta_{\omega}^{i}(x)\right)$. We remark that, by the transitivity of conditional measures, for $P$-a.e. $\omega$ one has $\mu_{(\omega, x)}^{i}=\left(\mu_{\omega}\right)_{x}^{\eta_{\omega}^{i}}$ for $\mu_{\omega}$-a.e. $x \in \Lambda_{i, \omega}:=\left\{x:(\omega, x) \in \Lambda_{i}\right\}$, where $\left(\mu_{\omega}\right)_{x}^{\eta_{\omega}^{i}}$ is the conditional measure of $\mu_{\omega}$ on $\eta_{\omega}^{i}(x)$. Let $d_{(\omega, x)}^{u, i}(\cdot, \cdot)$ be the metric on $W^{u, i}(\omega, x)$ induced by its inherited Riemannian structure and put for $\rho>0$

$$
B^{u, i}(\omega, x ; \rho)=\left\{y \in W^{u, i}(\omega, x): d_{(\omega, x)}^{u, i}(x, y)<\rho\right\} .
$$


Define

$$
\delta_{i}(\omega, x):=\lim _{\rho \rightarrow 0} \frac{\log \mu_{(\omega, x)}^{i}\left(B^{u, i}(\omega, x ; \rho)\right)}{\log \rho}
$$

provided the limit exists (the definition is independent of the choice of $\eta_{i}$ ), and call it the dimension of $\mu$ on the $W^{u, i}$-manifold at $(\omega, x)$.

The following theorem extends the generalized entropy formula of Ledrappier and Young 8 ] to $(F, \mu)$.

Theorem 2.1. Let the RDS F be as given above so that (2.1) holds. Let $\mu$ be an Finvariant measure. Then for $\mu$-a.e. $(\omega, x)$ the limit (2.3) exists for $1 \leq i \leq u(\omega, x)$ and the formula

$$
h_{\mu}(F)=\int \sum_{i: \lambda_{i}(\omega, x)>0} \lambda_{i}(\omega, x) \gamma_{i}(\omega, x) d \mu
$$

holds true, where $\gamma_{i}(\omega, x):=\delta_{i}(\omega, x)-\delta_{i-1}(\omega, x)$ for $i=1, \cdots, u(\omega, x)$ and $\delta_{0}(\omega, x)$ $:=0$.

A detailed proof of Theorem 2.1] is given in 13 for i.i.d. random diffeomorphisms, more precisely, for the case where $\Omega=\operatorname{Diff}^{2}(M)^{\mathbf{Z}}, \theta$ is the left shift operator on $\Omega$, $P=\nu^{\mathbf{Z}}$ for some probability $\nu$ on $\operatorname{Diff}^{2}(M), F: \omega=\left(\cdots, \omega_{-1}, \omega_{0}, \omega_{1}, \cdots\right) \mapsto \omega_{0}$, and $\mu$ is a forward Markov $F$-invariant measure (i.e., $\mu_{\omega}$ depends only on the past $\left(\cdots, \omega_{-2}, \omega_{-1}\right)$ for $P$-a.e. $\omega$; see Arnold [1, Theorems 1.7.2 and 2.1.8] or Liu [9, Proposition 1.2] for properties of such measures). The proof of the present more general theorem is the same except that two modifications are needed. One is the arguments presented in Bahnmüller and Liu 2, Section 3]. We remark that $\operatorname{Diff}^{2}(M)$ is separable, and thus we can apply Lusin's theorem. The authors do not know if $\operatorname{Diff}^{2}(M)$ could be replaced with $\operatorname{Diff}^{1+\alpha}(M)$ by a suitable trick, noting that $\operatorname{Diff}^{1+\alpha}(M)$ is in general not separable. The other is the treatment in 2, Section 5], together with the following ergodic decomposition facts of $(F, \mu)$. First assume that $(\theta, P)$ is ergodic. Then, by a combination of the ergodic decomposition theorems of [15] and [5], there exists a measurable partition $\zeta$ of $(\Omega \times M, \mu)$ (with the $\mu$-completion of $\mathcal{F} \times \mathcal{B})$ such that, for $\mu_{\zeta}$-a.e. $C \in \zeta, \Theta^{-1} C=C,\left.\Theta\right|_{C}:\left(C, \mu_{C}\right) \hookleftarrow$ is ergodic and $\mu_{C}$ has marginal $P$ on $\Omega$, where $\mu_{C}$ is the conditional measure of $\mu$ on $C$. If $(\theta, P)$ is not ergodic, one takes a measurable partition $\Pi$ of $(\Omega, P)$ (with the $P$-completion of $\mathcal{F})$ such that $\theta^{-1} \Gamma=\Gamma,\left.\theta\right|_{\Gamma}:\left(\Gamma, P_{\Gamma}\right) \hookleftarrow$ is ergodic for $P_{\Pi}$-a.e. $\Gamma \in \Pi$. Let $\mu_{\pi^{-1} \Gamma}$ be the conditional measure of $\mu$ on $\pi^{-1} \Gamma$ (defined by considering the partition $\pi^{-1} \Pi$ of $\left.(\Omega \times M, \mu)\right)$. Then, for $P_{\Pi^{-}}$-a.e. $\Gamma \in \Pi, \mu_{\pi^{-1} \Gamma}$ is $\Theta$-invariant, and it has marginal $P_{\Gamma}$ on $\Omega$. To prove Theorem 2.1, it is enough to consider $\Theta:\left(\pi^{-1} \Gamma, \mu_{\pi^{-1} \Gamma}\right) \hookleftarrow$ for $P_{\Pi^{-a}}$ a.e. $\Gamma \in \Pi$ and to use arguments similar to 2, Section $5]$.

By considering $\Theta^{-1}:(\Omega \times M, \mu) \hookleftarrow$, Theorem 2.1 can also be formulated by means of the negative Lyapunov exponents. That is, letting $(F, \mu)$ be as given in Theorem 2.1 and letting $0>\lambda_{s(\omega, x)}(\omega, x)>\cdots>\lambda_{r(\omega, x)}(\omega, x)>-\infty$ be all the negative exponents of $F$ at $(\omega, x)$ (if there are any), then one has

$$
h_{\mu}(F)=\int \sum_{j: \lambda_{j}(\omega, x)<0}-\lambda_{j}(\omega, x) \gamma_{j}(\omega, x) d \mu,
$$

where $\gamma_{j}(\omega, x):=\delta_{j}(\omega, x)-\delta_{j+1}(\omega, x)$ for $s(\omega, x) \leq j \leq r(\omega, x)$ with the convention $\delta_{r(\omega, x)+1}(\omega, x):=0$, and $\delta_{j}(\omega, x)$ is the dimension of $\mu$ on the $W^{s, j}$-manifold at 
$(\omega, x)$ defined analogously to the unstable manifolds case by replacing $W^{u, i}(\omega, x)$ with the $C^{1,1}$ immersed submanifold

$$
W^{s, j}(\omega, x):=\left\{y \in M: \varlimsup_{n \rightarrow+\infty} \frac{1}{n} \log d\left(f_{\omega}^{n} x, f_{\omega}^{n} y\right) \leq \lambda_{j}(\omega, x)\right\} .
$$

We remark that $W^{s, s(\omega, x)}(\omega, x)$ coincides with

$$
W^{s}(\omega, x):=\left\{y \in M: \varlimsup_{n \rightarrow+\infty} \frac{1}{n} \log d\left(f_{\omega}^{n} x, f_{\omega}^{n} y\right)<0\right\},
$$

which is called the stable manifold of $F$ at $(\omega, x)$. For $\mu$-a.e. $(\omega, x)$, define $W^{s}(\omega, x)$ $=\{x\}$ if there are no negative exponents at $(\omega, x)$.

Let $\eta^{s}$ (resp. $\left.\eta^{u}\right)$ be a measurable partition of $(\Omega \times M, \mu)$ subordinate to the $W^{s}$-manifolds (resp. $W^{u}$-manifolds) of $(F, \mu)$ and finer than $\{\{\omega\} \times M: \omega \in \Omega\}$. Let $\mu_{(\omega, x)}^{s}$ be the conditional measure of $\mu$ on $\eta^{s}(\omega, x)=:\{\omega\} \times \eta_{\omega}^{s}(x)$ (identified with $\left.\eta_{\omega}^{s}(x)\right)$. Recall that for $P$-a.e. $\omega$ one has $\mu_{(\omega, x)}^{s}=\left(\mu_{\omega}\right)_{x}^{\eta_{\omega}^{s}}$ for $\mu_{\omega}$-a.e. $x \in M$, where $\left(\mu_{\omega}\right)_{x}^{\eta_{\omega}^{s}}$ is the conditional measure of $\mu_{\omega}$ on $\eta_{\omega}^{s}(x)$. Let $d_{(\omega, x)}^{s}(\cdot, \cdot)$ be the distance along $W^{s}(\omega, x)$ and for $\rho>0$ put

$$
B^{s}(\omega, x ; \rho)=\left\{y \in W^{s}(\omega, x): d_{(\omega, x)}^{s}(x, y)<\rho\right\} .
$$

Define $\mu_{(\omega, x)}^{u}$ and $B^{u}(\omega, x ; \rho)$ similarly. Then, as asserted above, the limits

$$
\begin{aligned}
d_{\mu_{\omega}}^{s}(x) & :=\lim _{\rho \rightarrow 0} \frac{\log \mu_{(\omega, x)}^{s}\left(B^{s}(\omega, x ; \rho)\right)}{\log \rho}, \\
d_{\mu_{\omega}}^{u}(x) & :=\lim _{\rho \rightarrow 0} \frac{\log \mu_{(\omega, x)}^{u}\left(B^{u}(\omega, x ; \rho)\right)}{\log \rho}
\end{aligned}
$$

exist $\mu$ almost everywhere. As functions of $(\omega, x)$, they are $\Theta$-invariant and hence, when $\mu$ is ergodic, $d_{\mu_{\omega}}^{s}(x)=d^{s}$ and $d_{\mu_{\omega}}^{u}(x)=d^{u} \mu$ almost everywhere for some constants $d^{s}$ and $d^{u}$. These facts play a crucial role in the proof of the main result stated in the next section.

\section{MAIN THEOREM}

An invariant measure $\mu$ of $F$ is said to be hyperbolic if all the Lyapunov exponents of $F$ are nonzero $\mu$ almost everywhere. In the rest of the paper, $\eta^{s}$ will be a measurable partition of $(\Omega \times M, \mu)$ subordinate to the $W^{s}$-manifolds of $(F, \mu)$ such that $\eta^{s} \geq \sigma:=\{\{\omega\} \times M: \omega \in \Omega\}, \Theta \eta^{s} \geq \eta^{s}, \Theta^{n} \eta^{s}$ tends to the partition into single points as $n \rightarrow+\infty$, and $H\left(\Theta \eta^{s} \mid \eta^{s}\right)=h_{\mu}(F) ; \eta^{u}$ will be a measurable partition of $(\Omega \times M, \mu)$ subordinate to the $W^{u}$-manifolds of $(F, \mu)$ such that $\eta^{u} \geq \sigma$, $\Theta^{-1} \eta^{u} \geq \eta^{u}, \Theta^{-n} \eta^{s}$ tends to the partition into single points, and $H\left(\Theta^{-1} \eta^{u} \mid \eta^{u}\right)=$ $h_{\mu}(F)$ (see [11, Chapter VI] for their existence).

The main result of this paper is the following theorem.

Theorem 3.1. Let the RDS F be as given above so that (2.1) holds. Let $\mu$ be an $F$-invariant measure. If $\mu$ is hyperbolic and ergodic, then the following properties hold:

(1) for every $\varepsilon>0$, there exists a set $\Lambda \subset \Omega \times M$ with $\mu(\Lambda)>1-\varepsilon$ and constants $\kappa \geq 1, \rho_{0}>0$ such that for every $(\omega, x) \in \Lambda$ and every $\rho \in\left(0, \rho_{0}\right)$,

$$
\begin{array}{r}
\rho^{\varepsilon} \mu_{(\omega, x)}^{s}\left(B^{s}\left(\omega, x ; \frac{\rho}{\kappa}\right)\right) \mu_{(\omega, x)}^{u}\left(B^{u}\left(\omega, x ; \frac{\rho}{\kappa}\right)\right) \leq \mu_{\omega}(B(x, \rho)) \\
\leq \rho^{-\varepsilon} \mu_{(\omega, x)}^{s}\left(B^{s}(\omega, x ; \kappa \rho)\right) \mu_{(\omega, x)}^{u}\left(B^{u}(\omega, x ; \kappa \rho)\right) ;
\end{array}
$$


(2) for P-a.e. w the limit

$$
d_{\mu_{\omega}}(x):=\lim _{\rho \rightarrow 0} \frac{\log \mu_{\omega}(B(x, \rho))}{\log \rho}
$$

exists for $\mu_{\omega}$-a.e. $x \in M$ and

$$
d_{\mu_{\omega}}(x)=d^{s}+d^{u}, \quad \mu \text {-a.e. }(\omega, x) \in \Omega \times M .
$$

When $\mu$ is not ergodic, the limit (3.2) still exists and

$$
d_{\mu_{\omega}}(x)=d_{\mu_{\omega}}^{s}(x)+d_{\mu_{\omega}}^{u}(x)
$$

for $\mu$-a.e. $(\omega, x) \in \Omega \times M$.

Let $F$ be an RDS generated by i.i.d. random diffeomorphisms introduced in the paragraph after Theorem 2.1] It naturally induces a family of Markov processes with transition probabilities $Q(x, \cdot), x \in M$, given by

$$
Q(x, A):=P\left\{\omega: f_{\omega} x \in A\right\}=\nu\{f: f x \in A\} \text { for Borel } A \subset M .
$$

A probability $\rho$ on $M$ is called a stationary measure of $F$ if $\int Q(x, A) d \rho(x)=\rho(A)$ for all Borel $A \subset M$, or equivalently $\int f \rho d \nu(f)=\rho$. Such measures are of particular interest (see Kifer [7]), and they correspond in a one-to-one way to the forward Markov invariant measures of $F$, with the correspondence being given by

$$
\rho \mapsto \rho^{*} \text { with } \rho_{\omega}^{*}:=\lim _{n \rightarrow+\infty} f_{\theta^{-n} \omega}^{n} \rho, P \text {-a.e. } \omega
$$

and

$$
\rho^{*} \mapsto \rho:=\int \rho_{\omega}^{*} d P(\omega)
$$

(see, e.g., 9]). We remark that, even when $d_{\rho}(x)=d \rho$-a.e. $x$ and $d_{\rho_{\omega}^{*}}(x)=d^{*}$ $\rho^{*}$-a.e. $(\omega, x)$ for some constants $d$ and $d^{*}$, one may have $d \neq d^{*}$. This can be seen from the following example. Let $f \in \operatorname{Diff}^{1}(M)$ and let $x_{0}$ be a sink of $f$. Let $\nu$ be a probability on $\operatorname{Diff}^{1}(M)$ such that it is supported by a neighborhood $\mathcal{U}(f)$ of $f$ in $\operatorname{Diff}^{1}(M)$ and that the transition probabilities $Q(x, \cdot), x \in M$, have a density with respect to the Lebesgue measure on $M$ (see [11, Section IV.1] for the definition). When $\mathcal{U}(f)$ is sufficiently small, there is $\varepsilon_{0}>0$ such that for each $\omega \in \mathcal{U}(f)^{\mathbf{Z}}$ there is a unique $x_{\omega} \in M$ such that $d\left(f_{\omega}^{n} x_{\omega}, x_{0}\right)<\varepsilon_{0}$ for all $n \in \mathbf{Z}$. In this case, by [10, there is a unique stationary measure $\rho$ of $F$, it is absolutely continuous with respect to the Lebesgue measure on $M$, and $\rho_{\omega}^{*}=\delta_{x_{\omega}}$ for $P$-a.e. $\omega$. Clearly $d_{\rho}(x)=\operatorname{dim} M$ $\rho$-a.e. $x$ and $d_{\rho_{\omega}^{*}}(x)=0 \rho^{*}$-a.e. $(\omega, x)$.

\section{Reconstruction of a special partition}

Let $F$ be as given in Theorem 3.1 and let $\mu$ be an $F$-invariant measure. For a measurable partition $\xi$ of $(\Omega \times M, \mu)$ and for all integers $k, l \geq 0$, we define $\xi_{k}^{l}:=\bigvee_{j=-k}^{l} \Theta^{-j} \xi$ and put $\xi^{+}:=\xi_{0}^{\infty}$ and $\xi^{-}:=\xi_{\infty}^{0}$. Also write $\sigma:=\pi^{-1} \mathcal{F}$.

We have the following lemma, which describes the exponential decay of the distance of two initial points whenever their orbits stay close enough in a long time interval $[-n,+n]$.

Lemma 4.1. Suppose that $\left|\lambda_{j}(\omega, x)\right| \geq \lambda>0,1 \leq j \leq r(\omega, x)$, for $\mu$-a.e. $(\omega, x)$ and for a constant $\lambda>0$. Let $\left\{\Phi_{(\omega, x)}\right\}$ be a system of $(\varepsilon, \ell)$-charts introduced in 13] (where $0<\varepsilon<\frac{1}{200} \min \{\lambda, 1\}$ ) and let $H_{(\omega, x)}^{n}:=\Phi_{\Theta^{n}(\omega, x)}^{-1} \circ f_{\omega}^{n} \circ \Phi_{(\omega, x)}, n \in \mathbf{Z}$, 
be defined wherever they make sense. Then for any $0<\delta \leq 1$, the following holds true for $\mu$-a.e. $(\omega, x)$ : if $z=\Phi_{(\omega, x)}^{-1} y \in \overline{\mathbf{R}}\left(\ell(\omega, x)^{-1}\right)$ satisfies

$$
\left\|H_{(\omega, x)}^{k} z\right\| \leq \delta \ell\left(\Theta^{k}(\omega, x)\right)^{-1} \quad \text { for all }|k| \leq n,
$$

then

and therefore

$$
\|z\| \leq \delta \ell(\omega, x)^{-1} e^{-n(\lambda-3 \varepsilon)}
$$

$$
d(x, y) \leq 2 K_{0} \delta e^{-n(\lambda-3 \varepsilon)},
$$

where $K_{0}$ is a universal constant introduced in [13, Prop. 3.1].

A proof of the lemma can be carried out by employing Lyapunov charts (see 13 , Proposition 3.1]) and then using arguments similar to the uniformly hyperbolic case (see [10, Lemma 1.2]). The details are omitted here.

We will need a measurable partition $\alpha$ of $(\Omega \times M, \mu)$ so that it has finite conditional entropy $H_{\mu}(\alpha \mid \sigma)<+\infty$ and the diameter of $\left[\alpha_{n}^{n}\right]_{\omega}(x)$ decays exponentially fast as $n$ increases for $\mu$-a.e. $(\omega, x)$. But the partition $\alpha$ constructed in [13, Lemma 4.3] (generalizing the corresponding construction for a deterministic diffeomorphism in Ledrappier and Young [8) cannot be guaranteed to have these properties. In what follows we reconstruct such a partition. The treatment depends heavily on the previous work Qian et al. 13, to which we refer the reader for related notions and arguments.

In the rest of this section we assume that $\mu$ is ergodic and hyperbolic. Let $\lambda:=\min \left\{\left|\lambda_{i}\right|: i=1, \cdots, r\right\}$. Let $\left\{\Phi_{(\omega, x)}\right\}$ be a system of $(\varepsilon, \ell)$-charts introduced in [13, Sect. 3] with $0<\varepsilon<\frac{1}{200} \min \{\lambda, 1\}$. Let $l_{0}>0$ be a number so that the set

$$
\Delta:=\left\{(\omega, x): \ell(\omega, x) \leq l_{0}\right\}
$$

has positive $\mu$-measure. Define $n_{+}, n_{-}, n^{+}$and $n^{-}: \Delta \rightarrow \mathbf{Z}^{+}$by the following:

$$
\begin{aligned}
& n_{+}(\omega, x):=\inf \left\{n \geq 1: \Theta^{n}(\omega, x) \in \Delta\right\}, \\
& n_{-}(\omega, x):=\inf \left\{n \geq 1: \Theta^{-n}(\omega, x) \in \Delta\right\}, \\
& n^{+}(\omega, x):=\inf \left\{n \geq 0: \Theta^{n}(\omega, x) \in \Delta\right\}, \\
& n^{-}(\omega, x):=\inf \left\{n \geq 0: \Theta^{-n}(\omega, x) \in \Delta\right\} .
\end{aligned}
$$

Then let $\psi: \Omega \times M \rightarrow \mathbf{R}$ be given by

$$
\psi(\omega, x)= \begin{cases}\frac{\delta}{2 K_{0} l_{0}^{2}} e^{-2\left(\lambda_{0}+\varepsilon\right) \max \left\{n_{+}(\omega, x), n_{-}(\omega, x)\right\}}, & \text { if }(\omega, x) \in \Delta, \\ \frac{\delta}{2 K_{0} l_{0}^{2}}, & \text { otherwise }\end{cases}
$$

(where $0<\delta<1$ ), which is a modification of the corresponding definition in 13 , Sect. 4] by replacing $\widehat{S}_{u}$ with $\Delta$ and by doubling the exponent of the exponential term in the expression of $\psi$ (such a modification will accelerate the decay of the diameter of $\left[\alpha_{n}^{n}\right]_{\omega}(x)$ in $n$ for the partition $\alpha$ constructed below).

Let $U_{k}:=\left\{(\omega, x): e^{-k-1}<\psi(\omega, x) \leq e^{-k}\right\}, k \in \mathbf{Z}^{+}$. For each $k$ we choose a maximal $\frac{1}{2} e^{-k-1}$-separated subset $E_{k}$ of $M$ and define a partition $\beta_{k}$ of $M$ such that $\beta_{k}(x) \subset \overline{\operatorname{int}\left(\beta_{k}(x)\right)}$ and

$$
\operatorname{int}\left(\beta_{k}(x)\right)=\left\{y: d(x, y)<d(z, y) \text { for any } z \in E_{k} \backslash\{x\}\right\}
$$

for all $x \in E_{k}$, where $\operatorname{int}(A)$ denotes the interior of a set $A \subset M$. We then define a countable measurable partition $\alpha$ of $\Omega \times M$ by

$$
\alpha(\omega, x):=U_{k} \cap\left[\Omega \times \beta_{k}(x)\right] \text {, if }(\omega, x) \in U_{k} \text { for some } k \geq 0 .
$$


Since, due to Kac's lemma, $-\log \psi(\omega, x) \in L^{1}(\mu)$, by [11, Lemma VI.5.2], the partition $\alpha$ has finite conditional entropy $H_{\mu}(\alpha \mid \sigma)<+\infty$ and satisfies for $\mu$-a.e. $(\omega, x)$

$$
\alpha_{\omega}(x) \subset B(x, \psi(\omega, x)) .
$$

Besides those described in [13, Lemmas 4.3 and 5.2], the partition $\alpha$ has the following additional properties given in Lemmas 4.2 and 4.3 , where $H_{(\omega, x)}^{k}$ is as introduced in Lemma 4.1 and $\psi_{+}: \Omega \times M \rightarrow \mathbf{Z}^{+}$is defined by

$$
\psi_{+}(\omega, x):= \begin{cases}\frac{1}{2 K_{0} l_{0}^{2}} e^{-\left(\lambda_{0}+\varepsilon\right) n_{+}(\omega, x)}, & \text { if }(\omega, x) \in \Delta, \\ \frac{1}{2 K_{0} l_{0}^{2}}, & \text { otherwise. }\end{cases}
$$

Lemma 4.2. Let $(\omega, x) \in \Delta$.

(1) If $z \in \overline{\mathbf{R}}\left(\ell(\omega, x)^{-1}\right)$ satisfies $\|z\| \leq \delta l_{0} \psi_{+}(\omega, x)$, then

$$
\left\|H_{(\omega, x)}^{k} z\right\| \leq \delta \ell\left(\Theta^{k}(\omega, x)\right)^{-1}, \quad k=0,1, \cdots, n_{+}(\omega, x) .
$$

(2) If $y \in\left[\alpha_{0}^{n}\right]_{\omega}(x)$ for some $n \geq 0$, then

$$
\begin{aligned}
& \left\|H_{(\omega, x)}^{k} z\right\| \leq \delta \ell\left(\Theta^{k}(\omega, x)\right)^{-1}, \quad k=0,1, \cdots, n, \\
& \text { where } z=\Phi_{(\omega, x)}^{-1} y .
\end{aligned}
$$

Lemma 4.3. For $\mu$-a.e. $(\omega, x)$, if $y \in\left[\alpha_{0}^{n}\right]_{\omega}(x)$ for some $n \geq n^{+}(\omega, x)$, then

$$
\left\|H_{(\omega, x)}^{k} z\right\| \leq \delta \ell\left(\Theta^{k}(\omega, x)\right)^{-1}, \quad k=0,1, \cdots, n,
$$

where $z=\Phi_{(\omega, x)}^{-1} y$.

The proofs of Lemmas 4.2 and 4.3 follow the main lines of those of Claims 1-3 in [13, Sect. 4] (see also [8, Lemmas 9.3.2 and 9.3.3]). We present them below for completeness. For simplicity of notation, we will write $n_{+}=n_{+}(\omega, x)$ and $n^{+}=n^{+}(\omega, x)$ in the rest of this section.

Proof of Lemma 4.2, (1) From our assumption on $z$ and [13, Prop. 3.5 (1)], it follows that $H_{(\omega, x)}^{j} z \in \overline{\mathbf{R}}\left(\ell\left(\Theta^{j}(\omega, x)\right)^{-1}\right.$ ) and (note that $\lambda_{0} \geq \lambda_{1}+2 \varepsilon$ )

$$
\left\|H_{(\omega, x)}^{j} z\right\| \leq\|z\| e^{j \lambda_{0}} \text { for } j>0
$$

provided that $\|z\| e^{k \lambda_{0}} \leq \delta \ell\left(\Theta^{k}(\omega, x)\right)^{-1} e^{-\lambda_{1}-3 \varepsilon}$ for all $0 \leq k<j$. This is guaranteed for $0 \leq j \leq n_{+}$. Hence the desired conclusion follows from [13, Prop. 3.5 (1)].

(2) First observe that, if $y \in \alpha_{\omega}(x)$, then by [13, Prop. 3.1]

$$
z=\Phi_{(\omega, x)}^{-1} y \in \overline{\mathbf{R}}\left(\delta l_{0} \psi_{+}(\omega, x)\right)
$$

since $\ell(\omega, x) \leq l_{0}$ and $\psi(\omega, x) \leq \delta \psi_{+}(\omega, x)$. Therefore we have the desired conclusion for $0 \leq j \leq n_{+}$. Furthermore, if $n>n_{+}$and $y \in\left[\alpha_{0}^{n}\right]_{\omega}(x)$, then

$$
f_{\omega}^{n_{+}} y \in \alpha_{\theta^{n}+\omega}\left(f_{\omega}^{n_{+}} x\right),
$$

and we can apply (1), with $(\omega, x)$ and $z$ being replaced by $\Theta^{n_{+}}(\omega, x)$ and $\Phi_{\Theta^{n}+(\omega, x)}^{-1}\left(f_{\omega}^{n_{+}} y\right)$, respectively. An inductive argument completes the proof of (2). 
Proof of Lemma 4.3. Consider a point $(\omega, x)$ with the property that $\Theta^{n}(\omega, x) \in \Delta$ infinitely often as $n \rightarrow \pm \infty$. The collection of such points is of full $\mu$-measure. Let $y \in\left[\alpha_{0}^{n^{+}}\right]_{\omega}(x)$. We claim that $f_{\omega}^{j} y \in \Phi_{\Theta^{j}(\omega, x)} \overline{\mathbf{R}}\left(\delta \ell\left(\Theta^{j}(\omega, x)\right)^{-1}\right)$ for $j=$ $0,1, \cdots, n^{+}$. To prove the lemma, it suffices to prove this claim, since, if $y \in$ $\left[\alpha_{0}^{n}\right]_{\omega}(x)$ for some $n \geq n^{+}$, we can apply Lemma 4.2 $(2)$ to $f_{\omega}^{n^{+}} y$ (note that $\Theta^{n_{+}}(\omega, x)$ $\in \Delta)$, and this yields that

$$
f_{\omega}^{j} y \in \Phi_{\Theta^{j}(\omega, x)} \overline{\mathbf{R}}\left(\delta \ell\left(\Theta^{j}(\omega, x)\right)^{-1}\right) \quad \text { for } j=n^{+}, \cdots, n .
$$

To prove this claim, let us assume that $(\omega, x) \notin \Delta$ and write $k=-n_{-}\left(\Theta^{n^{+}}(\omega, x)\right)$ $+n^{+}<0$, i.e., $k$ is the largest negative integer such that $\Theta^{k}(\omega, x) \in \Delta$. Note that $\alpha_{\theta^{n+}{ }_{\omega}}\left(f_{\omega}^{n^{+}} x\right)$ lies well inside the chart at $f_{\omega}^{n^{+}} x$ since $\Theta^{n^{+}}(\omega, x) \in \Delta$. Hence, by our choice of $\psi$, we can prove inductively that $f_{\omega}^{-j+n^{+}} y$ lies inside the chart at $f_{\omega}^{-j+n^{+}} x$ for $j=1,2, \cdots, n^{+}-k$. Therefore

$$
\begin{aligned}
\left\|\Phi_{\Theta^{k}(\omega, x)}^{-1} f_{\omega}^{k} y\right\| & =\left\|H_{\Theta^{1+k}(\omega, x)}^{-1} \Phi_{\Theta^{1+k}(\omega, x)}^{-1} f_{\omega}^{1+k} y\right\| \\
& \leq e^{\lambda_{0}}\left\|\Phi_{\Theta^{1+k}(\omega, x)}^{-1} f_{\omega}^{1+k} y\right\| \leq \cdots \\
& \leq e^{\left(n^{+}-k\right) \lambda_{0}}\left\|\Phi_{\Theta^{n+}(\omega, x)}^{-1} f_{\omega}^{n^{+}} y\right\| \\
& \leq e^{\left(n^{+}-k\right) \lambda_{0}} l_{0} \psi\left(\Theta^{n^{+}}(\omega, x)\right) \\
& \leq \delta l_{0} \psi_{+}\left(\Theta^{k}(\omega, x)\right),
\end{aligned}
$$

the last inequality being guaranteed by

$$
2 \max \left\{n_{+}\left(\Theta^{n^{+}}(\omega, x)\right), n_{-}\left(\Theta^{n^{+}}(\omega, x)\right)\right\}-\left(n^{+}-k\right) \geq n_{+}\left(\Theta^{k}(\omega, x)\right),
$$

where $n_{+}\left(\Theta^{k}(\omega, x)\right)=n_{-}\left(\Theta^{n^{+}}(\omega, x)\right)=n^{+}-k$ since $(\omega, x) \notin \Delta$. Then, applying Lemma $4.2(1)$ to $f_{\omega}^{k} y$ yields the desired conclusion, since we now have $n_{+}\left(\Theta^{k}(\omega, x)\right)+k=n^{+}$.

We can also apply the above arguments to the system $\Theta^{-1}:(\Omega \times M, \mu) \hookleftarrow$, assuring that the partition $\alpha$ has the following additional properties. For $\mu$-a.e. $(\omega, x)$, if $y \in\left[\alpha_{n}^{0}\right]_{\omega}(x)$ for some $n \geq n^{-}(\omega, x)$, one has

$$
\left\|H_{(\omega, x)}^{-k} z\right\| \leq \delta \ell\left(\Theta^{-k}(\omega, x)\right)^{-1} \quad \text { for } 0 \leq k \leq n,
$$

where $z=\Phi_{(\omega, x)}^{-1} y$. Then, by Lemma 4.3 and the above equation, for $\mu$-a.e. $(\omega, x)$ we have

$$
\left\|H_{(\omega, x)}^{k} z\right\| \leq \delta \ell\left(\Theta^{k}(\omega, x)\right)^{-1} \quad \text { for }|k| \leq n
$$

provided $\Phi_{(\omega, x)} z \in\left[\alpha_{n}^{n}\right]_{\omega}(x)$ for some $n \geq \max \left\{n^{+}(\omega, x), n^{-}(\omega, x)\right\}$. Hence, by Lemma 4.1, for $\mu$-a.e. $(\omega, x)$ we have

$$
\left[\alpha_{n}^{n}\right]_{\omega}(x) \subset B\left(x, 2 K_{0} \delta e^{-n(\lambda-3 \varepsilon)}\right)
$$

when $n$ is sufficiently large.

From the above arguments together with [13, Lemma 3.6], it is also clear that for $\mu$-a.e. $(\omega, x)$

$$
\alpha_{\omega}^{+}(x) \subset \Phi_{(\omega, x)} W_{(\omega, x), \delta}^{s}(x) \subset B^{s}\left(\omega, x ; 2 K_{0} \delta\right)
$$

and

$$
\alpha_{\omega}^{-}(x) \subset \Phi_{(\omega, x)} W_{(\omega, x), \delta}^{u}(x) \subset B^{u}\left(\omega, x ; 2 K_{0} \delta\right) .
$$

Summing up the above results, we have proved the following. 
Lemma 4.4. Suppose that $\left|\lambda_{j}\right| \geq \lambda>0,1 \leq j \leq r$ and fix $0<\delta \leq 1$ arbitrarily. Then there is a measurable partition $\alpha$ of $\Omega \times M$ with $\int H_{\mu_{\omega}}\left(\alpha_{\omega}\right) d P(\omega)<+\infty$ such that, for some measurable function $n_{0}: \Omega \times M \rightarrow \mathbf{Z}^{+}$, one has for $\mu$-a.e. $(\omega, x)$ and all $n \geq n_{0}(\omega, x)$

$$
\left[\alpha_{n}^{n}\right]_{\omega}(x) \subset B\left(x, 2 K_{0} \delta e^{-n \lambda / 2}\right)
$$

and

$$
\begin{array}{llll}
{\left[\eta^{s} \vee \alpha_{n}^{0}\right]_{\omega}(x)} & \subset B^{s}(\omega, x ; n, \delta), & {\left[\eta^{s} \vee \alpha_{n}^{0}\right]_{\omega}(x)} & \subset B^{s}\left(\omega, x ; 2 K_{0} \delta e^{-n \lambda / 2}\right), \\
{\left[\eta^{u} \vee \alpha_{0}^{n}\right]_{\omega}(x)} & \subset B^{u}(\omega, x ; n, \delta), & {\left[\eta^{u} \vee \alpha_{0}^{n}\right]_{\omega}(x)} & \subset B^{u}\left(\omega, x ; 2 K_{0} \delta e^{-n \lambda / 2}\right),
\end{array}
$$

where

$$
B^{s}(\omega, x ; n, \delta):=\left\{y \in W^{s}(\omega, x): d_{\Theta^{-k}(\omega, x)}^{s}\left(f_{\omega}^{-k} x, f_{\omega}^{-k} y\right)<\delta \text { for } 0 \leq k \leq n\right\}
$$

and $B^{u}(\omega, x ; n, \delta)$ is defined analogously. Moreover, for $\mu$-a.e. $(\omega, x)$ one has

$$
\alpha_{\omega}^{+}(x) \subset B^{s}\left(\omega, x ; 2 K_{0} \delta\right) \text { and } \alpha_{\omega}^{-}(x) \subset B^{u}\left(\omega, x ; 2 K_{0} \delta\right) .
$$

Below we present some further properties of the partition $\alpha$ given above which will play an important role in our proof of Theorem 3.1 .

Lemma 4.5. Let $\alpha$ be the partition (depending particularly on $0<\delta \leq 1$ ) given above. Then one has for $\mu$-a.e. $(\omega, x)$

$$
\begin{aligned}
\lim _{\delta \downarrow 0} \lim _{n \rightarrow+\infty}-\frac{1}{2 n} \log \mu_{\omega}\left(\left[\alpha_{n}^{n}\right]_{\omega}(x)\right) & =h_{\mu}(F), \\
\lim _{\delta \downarrow 0} \lim _{n \rightarrow+\infty}^{*}-\frac{1}{n} \log \mu_{(\omega, x)}^{s}\left(\left[\alpha_{n}^{0}\right]_{\omega}(x)\right) & =h_{\mu}(F), \\
\lim _{\delta \downarrow 0} \lim _{n \rightarrow+\infty}^{*}-\frac{1}{n} \log \mu_{(\omega, x)}^{s}\left(\left[\alpha_{n}^{n}\right]_{\omega}(x)\right) & =h_{\mu}(F), \\
\lim _{\delta \downarrow 0} \lim _{n \rightarrow+\infty}^{*}-\frac{1}{n} \log \mu_{(\omega, x)}^{u}\left(\left[\alpha_{0}^{n}\right]_{\omega}(x)\right) & =h_{\mu}(F), \\
\lim _{\delta \downarrow 0} \lim _{n \rightarrow+\infty}^{*}-\frac{1}{n} \log \mu_{(\omega, x)}^{u}\left(\left[\alpha_{n}^{n}\right]_{\omega}(x)\right) & =h_{\mu}(F), \\
\lim _{n \rightarrow+\infty}-\frac{1}{n} \log \mu_{(\omega, x)}^{s}\left(\left[\alpha_{0}^{n}\right]_{\omega}(x)\right) & =0, \\
\lim _{n \rightarrow+\infty}-\frac{1}{n} \log \mu_{(\omega, x)}^{u}\left(\left[\alpha_{n}^{0}\right]_{\omega}(x)\right) & =0,
\end{aligned}
$$

where the limits " $\lim _{n \rightarrow+\infty}^{*}$ " in (4.2) -(4.5) are understood as both " $\underline{\lim }_{n \rightarrow+\infty}$ " and " $\varlimsup_{n \rightarrow+\infty}$ ".

We remark here that (4.6) and (4.7) in fact hold true for any measurable partition $\alpha$ of finite conditional entropy $H_{\mu}(\alpha \mid \sigma)<+\infty$. These facts would be crucial in Section 8.

In order to prove the above lemma, we first give another lemma which is similar to [8, Lemma 9.3.1].

Lemma 4.6. Let $\alpha$ be a partition of $\Omega \times M$ with $H_{\mu}(\alpha \mid \sigma)<+\infty$. Then

$$
\lim _{n \rightarrow+\infty}-\frac{1}{n} \log \mu_{(\omega, x)}^{u}\left(\left[\left(\eta^{u} \vee \alpha\right)_{n}^{n}\right]_{\omega}(x)\right)=h_{\mu}(F) \quad \text { for } \mu \text {-a.e. }(\omega, x) .
$$


Proof. Put

$$
\begin{aligned}
I_{\mu}(\xi \mid \sigma)(\omega, x) & :=-\log \mu_{\omega}\left(\xi_{\omega}(x)\right), \\
I_{\mu}(\xi \mid \eta \vee \sigma)(\omega, x) & :=-\log \left(\mu_{\omega}\right)_{x}^{\eta_{\omega}}\left(\xi_{\omega}(x)\right)
\end{aligned}
$$

for any two measurable partitions $\xi, \eta$ of $\Omega \times M$.

Clearly $-\log \mu_{(\omega, x)}^{u}\left(\left[\left(\eta^{u} \vee \alpha\right)_{n}^{n}\right]_{\omega}(x)\right)=I_{\mu}\left(\left[\eta^{u} \vee \alpha\right]_{n}^{n} \mid \eta^{u}\right)(\omega, x)$ since $\eta^{u}>\sigma$. Also, we can write $\frac{1}{n} I_{\mu}\left(\left[\eta^{u} \vee \alpha\right]_{n}^{n} \mid \eta^{u}\right)(\omega, x)$ as

$$
\frac{1}{n} I_{\mu}\left(\left[\eta^{u} \vee \alpha\right]_{0}^{n} \mid \eta^{u}\right)(\omega, x)+\frac{1}{n} I_{\mu}\left(\left[\eta^{u} \vee \alpha\right]_{n}^{0} \mid\left[\eta^{u} \vee \alpha\right]_{0}^{n}\right)(\omega, x),
$$

whose first term, by [13, Lemma 4.2], tends to $H_{\mu}\left(\eta^{u} \mid \Theta \eta^{u}\right)=H_{\mu}\left(\Theta^{-1} \eta^{u} \mid \eta^{u}\right)=$ $h_{\mu}(F)$ for $\mu$-a.e. $(\omega, x)$. Hence it suffices to prove

$$
\lim _{n \rightarrow+\infty} \frac{1}{n} I_{\mu}\left(\left[\eta^{u} \vee \alpha\right]_{n}^{0} \mid\left[\eta^{u} \vee \alpha\right]_{0}^{n}\right)(\omega, x)=0
$$

for $\mu$-a.e. $(\omega, x)$. Clearly

$$
I_{\mu}\left(\left[\eta^{u} \vee \alpha\right]_{n}^{0} \mid\left[\eta^{u} \vee \alpha\right]_{0}^{n}\right)(\omega, x)=\sum_{k=0}^{n-1} I_{\mu}\left(\Theta \alpha \mid\left[\eta^{u} \vee \alpha\right]_{0}^{n+k}\right)\left(\Theta^{-k}(\omega, x)\right)
$$

Put $I_{n}(\omega, x):=I_{\mu}\left(\Theta \alpha \mid\left[\eta^{u} \vee \alpha\right]_{0}^{n}\right)(\omega, x)$ and $I^{*}(\omega, x):=\sup _{n \geq 1} I_{n}(\omega, x)$. One can prove that $\int I^{*}(\omega, x) d \mu \leq H_{\mu}(\alpha \mid \sigma)+1$ and that $\left\{I_{n}, \mathcal{B}\left(\Theta \alpha \vee\left[\eta^{u} \vee \alpha\right]_{0}^{n}\right)\right\}$ is a supermartingale. Since $\mathcal{B}\left(\Theta^{-n} \eta^{u}\right) \nearrow \mathcal{B}(\Omega \times M)$, one then knows that

$$
I_{n} \rightarrow 0 \quad \mu \text {-a.e. and in } L^{1} \text {. }
$$

Therefore (4.8) follows by a slight variant of Birkhoff ergodic theorem.

Proof of Lemma 4.5. We first claim that

$$
\begin{aligned}
h_{\mu}^{\sigma}(\Theta, \alpha) & =H_{\mu}\left(\Theta^{-1} \alpha \mid \alpha^{-} \vee \sigma\right), \\
h_{\mu}^{\sigma}\left(\Theta^{-1}, \alpha\right) & =H_{\mu}\left(\Theta \alpha \mid \alpha^{+} \vee \sigma\right) .
\end{aligned}
$$

In fact, by the definition of conditional entropy we have (noting that $\Theta^{ \pm 1} \sigma=\sigma$ and $\Theta \mu=\mu$ )

$$
\begin{aligned}
h_{\mu}^{\sigma}(\Theta, \alpha) & :=\lim _{n \rightarrow+\infty} \frac{1}{n} H_{\mu}\left(\alpha_{0}^{n} \mid \sigma\right) \\
& =\lim _{n \rightarrow+\infty} \frac{1}{n}\left[H_{\mu}(\alpha \mid \sigma)+\sum_{k=0}^{n-1} H_{\mu}\left(\Theta^{-1} \alpha \mid \alpha_{k}^{0} \vee \sigma\right)\right] \\
& =H_{\mu}\left(\Theta^{-1} \alpha \mid \alpha^{-} \vee \sigma\right) \\
& =H_{\mu}\left(\Theta^{-1} \alpha^{-} \mid \alpha^{-} \vee \sigma\right) .
\end{aligned}
$$

Hence (4.9) holds true. (4.10) can be proved in a similar way.

Now we turn to the proof of the lemma. Let us first prove (4.4). It is easy to see that

$$
-\log \mu_{(\omega, x)}^{u}\left(\left[\alpha_{0}^{n}\right]_{\omega}(x)\right) \leq-\log \mu_{(\omega, x)}^{u}\left(\left[\left(\alpha \vee \eta^{u}\right)_{0}^{n}\right]_{\omega}(x)\right) .
$$

Hence, by [13, Lemma 4.2], we have

$$
\varlimsup_{n \rightarrow+\infty}-\frac{1}{n} \log \mu_{(\omega, x)}^{u}\left(\left[\alpha_{0}^{n}\right]_{\omega}(x)\right) \leq H_{\mu}\left(\eta^{u} \mid \Theta \eta^{u}\right)=h_{\mu}(F) .
$$


On the other hand, by Lemma 4.4 one has

$$
\begin{aligned}
\lim _{\delta \downarrow 0} \lim _{n \rightarrow+\infty}-\frac{1}{n} \log \mu_{(\omega, x)}^{u}\left(\left[\alpha_{0}^{n}\right]_{\omega}(x)\right) & \geq \lim _{\delta \downarrow 0} \varliminf_{n \rightarrow+\infty}-\frac{1}{n} \log \mu_{(\omega, x)}^{u}\left(B^{u}(\omega, x ; n, \delta)\right) \\
& =\lim _{\delta \downarrow 0} \underline{h}_{u}(\omega, x ; \delta) \\
& =h_{\mu}(F),
\end{aligned}
$$

where the last two equalities follow from [13, Prop. 4.1]. Hence (4.4) holds true. The proof of (4.2) is similar.

Combining Lemmas 4.4, 4.6 and [13, Prop. 4.1], one can easily prove (4.3) and (4.5) in a similar way.

We now turn to the proof of (4.1). Obviously

$$
I_{\mu}\left(\alpha_{n}^{n} \mid \sigma\right)(\omega, x)=I_{\mu}\left(\alpha_{n}^{0} \mid \sigma\right)(\omega, x)+I_{\mu}\left(\alpha_{0}^{n} \mid \alpha_{n}^{0} \vee \sigma\right)(\omega, x) .
$$

By a random version of Shannon-McMillan-Breiman theorem (see 4]) we have for $\mu$-a.e. $(\omega, x)$

$$
\lim _{n \rightarrow+\infty} \frac{1}{n} I_{\mu}\left(\alpha_{n}^{0} \mid \sigma\right)(\omega, x)=h_{\mu}^{\sigma}\left(\Theta^{-1}, \alpha\right)=h_{\mu}^{\sigma}(\Theta, \alpha)
$$

(the second equality is a standard fact). Write

$$
I_{\mu}\left(\alpha_{0}^{n} \mid \alpha_{n}^{0} \vee \sigma\right)(\omega, x)=\sum_{k=0}^{n-1} I_{\mu}\left(\Theta^{-1} \alpha \mid \alpha_{n+k}^{0} \vee \sigma\right)\left(\Theta^{k}(\omega, x)\right) .
$$

Similar to the proof of Lemma 4.6, we have

$$
I_{\mu}\left(\Theta^{-1} \alpha \mid \alpha_{n}^{0} \vee \sigma\right)(\omega, x) \rightarrow I_{\mu}\left(\Theta^{-1} \alpha \mid \alpha^{-} \vee \sigma\right)(\omega, x)
$$

$\mu$-a.e. and in $L^{1}$ as $n \rightarrow \infty$. Hence a slight variant of Birkhoff ergodic theorem gives

$$
\begin{aligned}
\lim _{n \rightarrow+\infty} \frac{1}{n} I_{\mu}\left(\alpha_{0}^{n} \mid \alpha_{n}^{0} \vee \sigma\right)(\omega, x) & =\lim _{n \rightarrow+\infty} \frac{1}{n} \sum_{k=0}^{n-1} I_{\mu}\left(\Theta^{-1} \alpha \mid \alpha_{n+k}^{0} \vee \sigma\right)\left(\Theta^{k}(\omega, x)\right) \\
& =\int I_{\mu}\left(\Theta^{-1} \alpha \mid \alpha^{-} \vee \sigma\right)(\omega, x) d \mu \\
& =H_{\mu}\left(\Theta^{-1} \alpha \mid \alpha^{-} \vee \sigma\right)=h_{\mu}^{\sigma}(\Theta, \alpha)
\end{aligned}
$$

for $\mu$-a.e. $(\omega, x)$. (4.1) is then proved since diam $\alpha_{\omega} \rightarrow 0$ as $\delta \rightarrow 0$, and hence

$$
\lim _{\delta \downarrow 0} h_{\mu}^{\sigma}(\Theta, \alpha)=h_{\mu}(F) .
$$

Finally we prove (4.7). In fact we can write $-\frac{1}{n} \log \mu_{(\omega, x)}^{u}\left(\left[\alpha_{n}^{0}\right]_{\omega}(x)\right)$ as

$$
\frac{1}{n} I_{\mu}\left(\alpha_{n}^{0} \mid \eta^{u} \vee \sigma\right)(\omega, x)=\frac{1}{n} \sum_{k=0}^{n} I_{\mu}\left(\alpha \mid \Theta^{-k} \eta^{u} \vee \Theta^{-1} \alpha_{0}^{k-1}\right)\left(\Theta^{-k}(\omega, x)\right),
$$

where $\alpha_{0}^{-1}:=\{\Omega \times M\}$. Similarly we have

$$
I_{\mu}\left(\alpha \mid \Theta^{-n} \eta^{u} \vee \Theta^{-1} \alpha_{0}^{n-1}\right)(\omega, x) \rightarrow 0 \quad \mu \text {-a.e. and in } L^{1}
$$

as $n \rightarrow+\infty$, and then we have (4.7). (4.6) can be proved similarly. 
Let $0<\varepsilon<1$ be given sufficiently small and let $\alpha$ be the partition introduced above for a fixed sufficiently small $\delta=\delta_{*}>0$. The system of Lyapunov charts employed in the construction of the special partition $\alpha$ will be referred to (in Section 8$)$ as a system of $\left(\varepsilon_{*}, \ell_{*}\right)$-Lyapunov charts $\left(0<\varepsilon_{*} \leq \frac{1}{200} \min \{\lambda, 1\}\right)$ with the corresponding universal constant $K_{0}$ (given by [13, Prop. 3.1]) denoted by $K_{*}$. By (2.6), (2.7) and Lemmas 4.4 and 4.5, one can find a set $\Gamma \subset \Omega \times M$ of measure $\mu(\Gamma)>1-\varepsilon / 2$ together with an integer $n_{0}=n_{0}(\varepsilon) \geq 1$ and a number $C=C(\varepsilon)>1$ such that, for every $(\omega, x) \in \Gamma$ and any integer $n \geq n_{0}$, the following statements hold:

a) for all integers $k \geq 1$ we have

$$
\begin{aligned}
& C^{-1} e^{-k(h+\varepsilon)} \leq \mu_{\omega}\left(\left[\alpha_{0}^{k}\right]_{\omega}(x)\right) \leq C e^{-k(h-\varepsilon)}, \\
& C^{-1} e^{-k(h+\varepsilon)} \leq \mu_{\omega}\left(\left[\alpha_{k}^{0}\right]_{\omega}(x)\right) \leq C e^{-k(h-\varepsilon)}, \\
& C^{-1} e^{-k(h+\varepsilon)} \leq \mu_{(\omega, x)}^{s}\left(\left[\alpha_{k}^{0}\right]_{\omega}(x)\right) \leq C e^{-k(h-\varepsilon)}, \\
& C^{-1} e^{-k \varepsilon} \leq \mu_{(\omega, x)}^{s}\left(\left[\alpha_{0}^{k}\right]_{\omega}(x)\right) \leq 1, \\
& C^{-1} e^{-k(h+\varepsilon)} \leq \mu_{(\omega, x)}^{u}\left(\left[\alpha_{0}^{k}\right]_{\omega}(x)\right) \leq C e^{-k(h-\varepsilon)}, \\
& C^{-1} e^{-k \varepsilon} \leq \mu_{(\omega, x)}^{u}\left(\left[\alpha_{k}^{0}\right]_{\omega}(x)\right) \leq 1,
\end{aligned}
$$

where $h=h_{\mu}(F)$;

b) for all integers $k \geq 1$ we have

c)

d)

$$
\begin{aligned}
& {\left[\alpha_{a n}^{a n}\right]_{\omega}(x) \subset B\left(x, e^{-n}\right), \quad \Gamma_{\omega} \cap B\left(x, e^{-n}\right) \subset \alpha_{\omega}(x),} \\
& {\left[\eta^{s} \vee \alpha_{a n}^{0}\right]_{\omega}(x) \subset \quad B^{s}\left(\omega, x ; e^{-n}\right) \quad \subset \eta_{\omega}^{s}(x),} \\
& {\left[\eta^{u} \vee \alpha_{0}^{a n}\right]_{\omega}(x) \subset \quad B^{u}\left(\omega, x ; e^{-n}\right) \quad \subset \eta_{\omega}^{u}(x),}
\end{aligned}
$$

where $a$ is the integral part of $2\left(1+\lambda^{-1}\right)$. (4.22) -(4.24) are clearly guaranteed by Lemma 4.4.

Since the induced metrics on $W^{s}(\omega, x)$ and $W^{u}(\omega, x)$ are locally (i.e., in a neighborhood of $x$ ) equivalent to the metric on $M$, by increasing $n_{0}$ if necessary, we may also assume the following:

e) for each $(\omega, x) \in \Gamma$ and any $z, z^{\prime} \in B^{s}\left(\omega, x ; e^{-n_{0}}\right)$

$$
\begin{aligned}
& d\left(z, z^{\prime}\right) \leq d_{(\omega, x)}^{s}\left(z, z^{\prime}\right) \leq 2 d\left(z, z^{\prime}\right), \\
& d\left(z, z^{\prime}\right) \leq d_{(\omega, x)}^{u}\left(z, z^{\prime}\right) \leq 2 d\left(z, z^{\prime}\right) ;
\end{aligned}
$$


f) for every $(\omega, x) \in \Gamma$ and $n \geq n_{0}$

$$
\begin{array}{ll}
B\left(x, \frac{e^{-n}}{2}\right) \cap \eta_{\omega}^{s}(x) \subset \quad B^{s}\left(\omega, x ; e^{-n}\right) & \subset B\left(x, e^{-n}\right) \cap \eta_{\omega}^{s}(x), \\
B\left(x, \frac{e^{-n}}{2}\right) \cap \eta_{\omega}^{u}(x) \subset \quad B^{u}\left(\omega, x ; e^{-n}\right) & \subset B\left(x, e^{-n}\right) \cap \eta_{\omega}^{u}(x) .
\end{array}
$$

We will need the following slight variant of Borel density lemma 6].

Proposition 4.7. Let $A \subset \Omega \times M$ be a measurable set with $\mu(A)>0$. Then for $\mu$-almost every $(\omega, x) \in A$

$$
\lim _{\rho \rightarrow 0} \frac{\mu_{\omega}\left(B(x, \rho) \cap A_{\omega}\right)}{\mu_{\omega}(B(x, \rho))}=1 .
$$

Furthermore, for each $\delta>0$ there is a set $\Delta \subset A$ with $\mu(\Delta)>\mu(A)-\delta$ and a number $\rho^{*}$ such that for all $(\omega, x) \in \Delta$ and $0<\rho<\rho^{*}$ one has

$$
\mu_{\omega}\left(B(x, \rho) \cap A_{\omega}\right) \geq \frac{1}{2} \mu_{\omega}(B(x, \rho)) .
$$

Proof. Let $A^{\prime}$ be the measurable set consisting of those points in $A$ satisfying (4.29). By Borel density lemma (see [3, Proposition 3]), $\mu_{\omega}\left(A_{\omega}^{\prime}\right)=\mu_{\omega}\left(A_{\omega}\right)$ for each $\omega \in \Omega$ and hence $\mu(A)=\int \mu_{\omega}\left(A_{\omega}\right) d P(\omega)=\int \mu_{\omega}\left(A_{\omega}^{\prime}\right) d P(\omega)=\mu\left(A^{\prime}\right)$.

Using arguments similar to the proof of Proposition 4.7, one can choose an integer $n_{1} \geq n_{0}$ and a set $\widehat{\Gamma} \subset \Gamma$ with $\mu(\widehat{\Gamma})>1-\varepsilon$ such that, for every $n \geq n_{1}$ and $(\omega, x) \in \widehat{\Gamma}$,

$$
\begin{aligned}
\mu_{\omega}\left(B\left(x, e^{-n}\right) \cap \Gamma_{\omega}\right) & \geq \frac{1}{2} \mu_{\omega}\left(B\left(x, e^{-n}\right)\right), \\
\mu_{(\omega, x)}^{s}\left(B^{s}\left(\omega, x ; e^{-n}\right) \cap \Gamma_{\omega}\right) & \geq \frac{1}{2} \mu_{(\omega, x)}^{s}\left(B^{s}\left(\omega, x ; e^{-n}\right)\right), \\
\mu_{(\omega, x)}^{u}\left(B^{u}\left(\omega, x ; e^{-n}\right) \cap \Gamma_{\omega}\right) & \geq \frac{1}{2} \mu_{(\omega, x)}^{u}\left(B^{u}\left(\omega, x ; e^{-n}\right)\right) .
\end{aligned}
$$

Furthermore we can require that for every $n \geq n_{1}$ and $(\omega, x) \in \widehat{\Gamma}$

$$
\begin{aligned}
& \mu_{(\omega, x)}^{s}\left(\left[\alpha_{0}^{a n}\right]_{\omega}(x) \cap B^{s}\left(\omega, x ; e^{-n}\right) \cap \Gamma_{\omega}\right) \geq e^{-n\left(d^{s}+\varepsilon\right)}, \\
& \mu_{(\omega, x)}^{u}\left(\left[\alpha_{a n}^{0}\right]_{\omega}(x) \cap B^{u}\left(\omega, x ; e^{-n}\right) \cap \Gamma_{\omega}\right) \geq e^{-n\left(d^{u}+\varepsilon\right)} .
\end{aligned}
$$

The requirements (4.33) and (4.34) seem easy to be satisfied but actually their proofs are very much involved with the techniques presented in $[13$ and are somehow lengthy. We will postpone the proofs to Section 8 .

\section{Preparatory lemmas}

To be more accessible, we use arguments slightly different from the corresponding ones of [3]. Fix $(\omega, x) \in \Gamma$ and an integer $n \geq n_{0}$. We consider the following classes $\mathcal{R}_{n}, \mathcal{F}_{n}^{s}$ and $\mathcal{F}_{n}^{u}$ of elements, respectively, of the partitions $\left[\alpha_{a n}^{a n}\right]_{\omega},\left[\alpha_{a n}^{0}\right]_{\omega}$ and $\left[\alpha_{0}^{a n}\right]_{\omega}$ (we call their elements "rectangles"):

$$
\begin{aligned}
& \mathcal{R}_{n}:=\left\{\left[\alpha_{a n}^{a n}\right]_{\omega}(y) \subset \alpha_{\omega}(x):\left[\alpha_{a n}^{a n}\right]_{\omega}(y) \cap \Gamma_{\omega} \neq \emptyset\right\}, \\
& \mathcal{F}_{n}^{s}:=\left\{\left[\alpha_{a n}^{0}\right]_{\omega}(y) \subset \alpha_{\omega}(x):\left[\alpha_{a n}^{0}\right]_{\omega}(y) \cap \Gamma_{\omega} \neq \emptyset\right\}, \\
& \mathcal{F}_{n}^{u}:=\left\{\left[\alpha_{0}^{a n}\right]_{\omega}(y) \subset \alpha_{\omega}(x):\left[\alpha_{0}^{a n}\right]_{\omega}(y) \cap \Gamma_{\omega} \neq \emptyset\right\} .
\end{aligned}
$$


The rectangles in $\mathcal{R}_{n}$ carry all the $\mu_{\omega}$-measures of the set $\alpha_{\omega}(x) \cap \Gamma_{\omega}$, i.e.,

$$
\sum_{R \in \mathcal{R}_{n}} \mu_{\omega}\left(R \cap \Gamma_{\omega}\right)=\mu_{\omega}\left(\alpha_{\omega}(x) \cap \Gamma_{\omega}\right) .
$$

It is clear that the rectangles in $\mathcal{R}_{n}$ belong to $\mathcal{F}_{n}^{s} \vee \mathcal{F}_{n}^{u}:=\left\{R^{s} \cap R^{u}: R^{s} \in \mathcal{F}_{n}^{s}, R^{u} \in\right.$ $\left.\mathcal{F}_{n}^{u}, R^{s} \cap R^{u} \neq \emptyset\right\}$. But, besides these elements, there are in general other ones in $\mathcal{F}_{n}^{s} \vee \mathcal{F}_{n}^{u}$. That is, the sample measure $\mu_{\omega}$ conditioned on $\alpha_{\omega}(x) \cap \Gamma_{\omega}$ has a deviation from the "direct product structure" at the "level" $n$.

Following [3], we will compare the numbers of rectangles in $\mathcal{R}_{n}, \mathcal{F}_{n}^{s}$ and $\mathcal{F}_{n}^{u}$ intersecting a given set. This will allow us to evaluate the deviation of the sample measure $\mu_{\omega}$ from the "direct product structure" at the level $n$. For each $A \subset \alpha_{\omega}(x)$, we define a series of subsets of $\mathcal{R}_{n}, \mathcal{F}_{n}^{s}$ or $\mathcal{F}_{n}^{u}$ by the following:

$$
\begin{aligned}
N(n, A) & :=\left\{R \in \mathcal{R}_{n}: R \cap A \neq \emptyset\right\}, \\
N^{s}(n, y, A) & :=N\left(n, \eta_{\omega}^{s}(y) \cap \Gamma_{\omega} \cap A\right), \\
N^{u}(n, y, A) & :=N\left(n, \eta_{\omega}^{u}(y) \cap \Gamma_{\omega} \cap A\right), \\
\widehat{N}^{s}(n, A) & :=\left\{R \in \mathcal{F}_{n}^{s}: R \cap A \neq \emptyset\right\}, \\
\widehat{N}^{u}(n, A) & :=\left\{R \in \mathcal{F}_{n}^{u}: R \cap A \neq \emptyset\right\} .
\end{aligned}
$$

One should bear in mind that, though we omit writing $\alpha_{\omega}(x)$ 's in the notations, all the sets defined above depend on $\alpha_{\omega}(x)$. From here on we always denote by $\# A$ the cardinality of a countable set $A$.

Lemma 5.1. For each $(\omega, x) \in \Gamma$ and integer $n \geq n_{0}+1$, we have

$$
\begin{aligned}
& \# N^{s}\left(n, x, B\left(x, e^{-n}\right)\right) \leq \mu_{(\omega, x)}^{s}\left(B^{s}\left(\omega, x ; 4 e^{-n}\right)\right) \cdot C e^{a n(h+\varepsilon)}, \\
& \# N^{u}\left(n, x, B\left(x, e^{-n}\right)\right) \leq \mu_{(\omega, x)}^{u}\left(B^{u}\left(\omega, x ; 4 e^{-n}\right)\right) \cdot C e^{a n(h+\varepsilon)} .
\end{aligned}
$$

Proof. Let $R \in N^{s}\left(n, x, B\left(x, e^{-n}\right)\right)$ and let $z \in R \cap \eta_{\omega}^{s}(x) \cap B\left(x, e^{-n}\right) \cap \Gamma_{\omega}$. Then $R=\left[\alpha_{a n}^{a n}\right]_{\omega}(z)$. By (4.18) we have

$$
\mu_{(\omega, x)}^{s}(R)=\mu_{(\omega, z)}^{s}\left(\left[\alpha_{a n}^{a n}\right]_{\omega}(z)\right) \geq C^{-1} e^{-a n(h+\varepsilon)} .
$$

By (4.23) one has $R \cap \eta_{\omega}^{s}(x) \subset B^{s}\left(\omega, z ; e^{-n}\right)$. But, by (4.27), we have

$$
z \in \eta_{\omega}^{s}(x) \cap B\left(x, e^{-n}\right) \subset B^{s}\left(\omega, x ; e^{-n+1}\right) .
$$

Therefore

$$
R \cap \eta_{\omega}^{s}(x) \subset B^{s}\left(\omega, x ; e^{-n}+e^{-n+1}\right) \subset B^{s}\left(\omega, x ; 4 e^{-n}\right)
$$

and hence

$$
\mu_{(\omega, x)}^{s}\left(B^{s}\left(\omega, x ; 4 e^{-n}\right)\right) \geq \sum_{R \in N^{s}\left(n, x, B\left(x, e^{-n}\right)\right)} \mu_{(\omega, x)}^{s}(R) .
$$

This implies

$$
\mu_{(\omega, x)}^{s}\left(B^{s}\left(\omega, x ; 4 e^{-n}\right)\right) \geq \# N^{s}\left(n, x, B\left(x, e^{-n}\right)\right) \cdot C^{-1} e^{-a n(h+\varepsilon)} .
$$

The proof of the second inequality is similar.

Lemma 5.2. For each $(\omega, x) \in \widehat{\Gamma}$ and $n \geq n_{1}$, we have

$$
\mu_{\omega}\left(B\left(x, e^{-n}\right)\right) \leq \# N\left(n, B\left(x, e^{-n}\right) \cap \Gamma_{\omega}\right) \cdot 2 C e^{-2 a n(h-\varepsilon)} .
$$


Proof. In view of (4.30) we have

$$
\begin{aligned}
& \frac{1}{2} \mu_{\omega}\left(B\left(x, e^{-n}\right)\right) \leq \mu_{\omega}\left(B\left(x, e^{-n}\right) \cap \Gamma_{\omega}\right) \leq \sum_{R \in N\left(n, B\left(x, e^{-n}\right) \cap \Gamma_{\omega}\right)} \mu_{\omega}(R) \\
& \leq \# N\left(n, B\left(x, e^{-n}\right) \cap \Gamma_{\omega}\right) \cdot \max \left\{\mu_{\omega}(R): R \in N\left(n, B\left(x, e^{-n}\right) \cap \Gamma_{\omega}\right)\right\} .
\end{aligned}
$$

Let $R \in N\left(n, B\left(x, e^{-n}\right) \cap \Gamma_{\omega}\right)$. Since $R=\left[\alpha_{a n}^{a n}\right]_{\omega}(z)$ for a point $z \in R \cap B\left(x, e^{-n}\right) \cap$ $\Gamma_{\omega}$, the inequality in the lemma follows from (4.17).

We now estimate the numbers of rectangles in the classes $\mathcal{R}_{n}, \mathcal{F}_{n}^{s}$ and $\mathcal{F}_{n}^{u}$. Clearly we have the following lemma.

Lemma 5.3. For each $(\omega, x) \in \Gamma$ and each $n \geq n_{0}$,

$$
\# N\left(n, B\left(x, e^{-n}\right) \cap \Gamma_{\omega}\right) \leq \# \widehat{N}^{s}\left(n, B\left(x, e^{-n}\right) \cap \Gamma_{\omega}\right) \cdot \# \widehat{N}^{u}\left(n, B\left(x, e^{-n}\right) \cap \Gamma_{\omega}\right) .
$$

Our next goal is to compare the growth rate in $n$ of the number of rectangles in $\widehat{N}^{s}\left(n, B\left(x, e^{-n}\right) \cap \Gamma_{\omega}\right)\left(\right.$ resp. $\left.\widehat{N}^{u}\left(n, B\left(x, e^{-n}\right) \cap \Gamma_{\omega}\right)\right)$ with that of the number of rectangles in $N^{s}\left(n, x, B\left(x, e^{-n}\right)\right)$ (resp. $N^{u}\left(n, x, B\left(x, e^{-n}\right)\right)$ ). We start with the following auxiliary result.

Lemma 5.4. For each $(\omega, x) \in \Gamma$ and integer $n \geq n_{0}$, we have

$$
\begin{aligned}
& \# \widehat{N}^{s}\left(n, \alpha_{\omega}(x)\right) \leq C e^{a n(h+\varepsilon)}, \\
& \# \widehat{N}^{u}\left(n, \alpha_{\omega}(x)\right) \leq C e^{a n(h+\varepsilon)} .
\end{aligned}
$$

Proof. Since $\alpha_{\omega}(x)$ is the union of a collection of rectangles $R=\left[\alpha_{a n}^{0}\right]_{\omega}(z)$, we have

$$
1 \geq \mu_{\omega}\left(\alpha_{\omega}(x)\right) \geq \sum_{R \in \widehat{N}^{s}\left(n, \alpha_{\omega}(x)\right)} \mu_{\omega}(R) .
$$

Hence, by (4.12), we have

$$
\# \widehat{N}^{s}\left(n, \alpha_{\omega}(x)\right) \leq \frac{1}{\min \left\{\mu_{\omega}\left(\left[\alpha_{a n}^{0}\right]_{\omega}(z)\right): z \in \Gamma_{\omega} \cap \alpha_{\omega}(x)\right\}} \leq C e^{a n(h+\varepsilon)} .
$$

The second inequality in the lemma can be proved in a similar way.

The following two lemmas show that the cardinalities of $\widehat{N}^{s}\left(n, B\left(x, e^{-n}\right) \cap \Gamma_{\omega}\right)$ and $N^{s}\left(n, x, B\left(x, e^{-n}\right)\right)\left(\operatorname{resp} . \widehat{N}^{u}\left(n, B\left(x, e^{-n}\right) \cap \Gamma_{\omega}\right)\right.$ and $\left.N^{u}\left(n, x, B\left(x, e^{-n}\right)\right)\right)$ are of almost the same growth rate in $n$ up to a small exponent.

Lemma 5.5. For each $(\omega, x) \in \Gamma, y \in \alpha_{\omega}(x)$ and $n \geq n_{0}$, we have

$$
\begin{aligned}
& \# \widehat{N}^{s}\left(n, B\left(y, e^{-n}\right) \cap \Gamma_{\omega}\right) \geq \# N^{s}\left(n, y, B\left(y, e^{-n}\right)\right) \cdot C^{-2} e^{-2 a n \varepsilon}, \\
& \# \widehat{N}^{u}\left(n, B\left(y, e^{-n}\right) \cap \Gamma_{\omega}\right) \geq \# N^{u}\left(n, y, B\left(y, e^{-n}\right)\right) \cdot C^{-2} e^{-2 a n \varepsilon} .
\end{aligned}
$$

Proof. Obviously $\widehat{N}^{s}\left(n, \eta_{\omega}^{s}(y) \cap B\left(y, e^{-n}\right) \cap \Gamma_{\omega}\right) \subset \widehat{N}^{s}\left(n, B\left(y, e^{-n}\right) \cap \Gamma_{\omega}\right)$, hence

$$
\# \widehat{N}^{s}\left(n, \eta_{\omega}^{s}(y) \cap B\left(y, e^{-n}\right) \cap \Gamma_{\omega}\right) \leq \# \widehat{N}^{s}\left(n, B\left(y, e^{-n}\right) \cap \Gamma_{\omega}\right) .
$$

On the other hand, for each rectangle $R^{s} \in \widehat{N}^{s}\left(n, \eta_{\omega}^{s}(y) \cap B\left(y, e^{-n}\right) \cap \Gamma_{\omega}\right)$ we can find a point $z$ in $\eta_{\omega}^{s}(y) \cap B\left(y, e^{-n}\right) \cap \Gamma_{\omega}$ such that $R^{s}=\left[\alpha_{a n}^{0}\right]_{\omega}(z)$. For such $z$, $R=\left[\alpha_{a n}^{a n}\right]_{\omega}(z)$ is a rectangle in $N^{s}\left(n, y, B\left(y, e^{-n}\right)\right)$. For any fixed

$$
R^{s} \in \widehat{N}^{s}\left(n, \eta_{\omega}^{s}(y) \cap B\left(y, e^{-n}\right) \cap \Gamma_{\omega}\right),
$$


there are at most $A_{n}$ distinct rectangles $R$ in $N^{s}\left(n, y, B\left(y, e^{-n}\right)\right)$ with such a correspondence, where by (4.13) and (4.18)

$$
\begin{aligned}
A_{n} & \leq \frac{\mu_{(\omega, y)}^{s}\left(R^{s}\right)}{\min \left\{\mu_{(\omega, y)}^{s}(R): R \in N^{s}\left(n, y, B\left(y, e^{-n}\right)\right), R \subset R^{s}\right\}} \\
& =\frac{\mu_{(\omega, z)}^{s}\left(\left[\alpha_{a n}^{0}\right]_{\omega}(z)\right)}{\min \left\{\mu_{(\omega, y)}^{s}\left(\left[\alpha_{a n}^{a n}\right]_{\omega}\left(z^{\prime}\right)\right): z^{\prime} \in \eta_{\omega}^{s}(y) \bigcap B\left(y, e^{-n}\right) \bigcap R^{s} \bigcap \Gamma_{\omega}\right\}} \\
& \leq C e^{-a n(h-\varepsilon)} /\left[C^{-1} e^{-a n(h+\varepsilon)}\right]=C^{2} e^{2 a n \varepsilon} .
\end{aligned}
$$

Therefore

$$
\# \widehat{N}^{s}\left(n, \eta_{\omega}^{s}(y) \cap B\left(y, e^{-n}\right) \cap \Gamma_{\omega}\right) \geq \# N^{s}\left(n, y, B\left(y, e^{-n}\right)\right) \cdot C^{-2} e^{-2 a n \varepsilon} .
$$

This implies the first inequality in the lemma. The other one can be proved analogously.

Lemma 5.6. For $\mu_{\omega}$-a.e. $y \in \alpha_{\omega}(x) \cap \widehat{\Gamma}_{\omega}$, we have

$$
\begin{gathered}
\varlimsup_{n \rightarrow+\infty} \frac{\# \widehat{N}^{s}\left(n, B\left(y, e^{-n}\right) \cap \Gamma_{\omega}\right)}{\# N^{s}\left(n, y, B\left(y, e^{-n}\right)\right)} e^{-5 a n \varepsilon}=0, \\
\varlimsup_{n \rightarrow+\infty} \frac{\# \widehat{N}^{u}\left(n, B\left(y, e^{-n}\right) \cap \Gamma_{\omega}\right)}{\# N^{u}\left(n, y, B\left(y, e^{-n}\right)\right)} e^{-5 a n \varepsilon}=0 .
\end{gathered}
$$

Proof. Except for applying our Lemma 5.4 instead of [3, Lemma 4], the proof is the same as that of [3, Lemma 5]. We present it here for completeness. By (4.27) and (4.31), for each $y \in \widehat{\Gamma}_{\omega}$ and $n \geq n_{1}$, we have

$$
\mu_{(\omega, y)}^{s}\left(B\left(y, e^{-n}\right) \cap \Gamma_{\omega}\right) \geq \mu_{(\omega, y)}^{s}\left(B^{s}\left(\omega, y ; e^{-n}\right) \cap \Gamma_{\omega}\right) \geq \frac{1}{2} \mu_{(\omega, y)}^{s}\left(B^{s}\left(\omega, y ; e^{-n}\right)\right)
$$

and

$$
\mu_{(\omega, y)}^{s}\left(B\left(y, e^{-n}\right) \cap \Gamma_{\omega}\right) \leq \sum_{R \in N^{s}} \sum_{\left(n, y, B\left(y, e^{-n}\right)\right)} \mu_{(\omega, y)}^{s}(R) .
$$

Then, by (4.13), (4.18), (4.20) and noting $a>1$, one has

$$
\begin{aligned}
& \# N^{s}\left(n, y, B\left(y, e^{-n}\right)\right) \\
\geq & \frac{\mu_{(\omega, y)}^{s}\left(B\left(y, e^{-n}\right) \cap \Gamma_{\omega}\right)}{\max \left\{\mu_{(\omega, y)}^{s}\left(\left[\alpha_{a n}^{a n}\right]_{\omega}(z)\right): z \in \eta_{\omega}^{s}(y) \cap \alpha_{\omega}(x) \cap \Gamma_{\omega}\right\}} \\
\geq & \frac{1}{2} \cdot \frac{\mu_{(\omega, y)}^{s}\left(B^{s}\left(\omega, y ; e^{-n}\right)\right)}{\max \left\{\mu_{(\omega, z)}^{s}\left(\left[\alpha_{a n}^{a n}\right]_{\omega}(z)\right): z \in \eta_{\omega}^{s}(y) \cap \alpha_{\omega}(x) \cap \Gamma_{\omega}\right\}} \\
\geq & \frac{1}{2 C} \frac{e^{-n\left(d^{s}+\varepsilon\right)}}{e^{-a n(h-\varepsilon)}} \geq \frac{1}{2 C} e^{-n\left(d^{s}-a h+2 a \varepsilon\right)} .
\end{aligned}
$$

For any integer $k \geq 1$, we consider the set

$$
F_{k}:=\left\{y \in \alpha_{\omega}(x) \cap \widehat{\Gamma}_{\omega}: \varlimsup_{n \rightarrow+\infty} \frac{\# \widehat{N}^{s}\left(n, B\left(y, e^{-n}\right) \cap \Gamma_{\omega}\right)}{\# N^{s}\left(n, y, B\left(y, e^{-n}\right)\right)} e^{-5 a n \varepsilon} \geq \frac{1}{k}\right\} .
$$


For each $y \in F_{k}$ take an increasing sequence $\left\{m_{j}=m_{j}(y) \geq n_{1}\right\}_{j=1}^{\infty}$ of positive integers such that for $n=m_{j}, j=1,2, \cdots$,

$$
\begin{aligned}
\# \widehat{N}^{s}\left(n, B\left(y, e^{-n}\right) \cap \Gamma_{\omega}\right) & \geq \frac{1}{2 k} \# N^{s}\left(n, y, B\left(y, e^{-n}\right)\right) e^{5 a n \varepsilon} \\
& \geq \frac{1}{4 k C} e^{-n\left(d^{s}-a h-3 a \varepsilon\right)} .
\end{aligned}
$$

We wish to show that $\mu_{\omega}\left(F_{k}\right)=0$. Assume on the contrary that $\mu_{\omega}\left(F_{k}\right)>0$. Let $F_{k}^{\prime} \subset F_{k}$ be the set of points $z \in F_{k}$ for which there exists the limit

$$
\lim _{\rho \rightarrow 0} \frac{\log \mu_{(\omega, z)}^{s}\left(B^{s}(\omega, z ; \rho)\right)}{\log \rho}=d^{s} .
$$

Clearly $\mu_{\omega}\left(F_{k}^{\prime}\right)=\mu_{\omega}\left(F_{k}\right)>0$. Then we can find a point $y \in F_{k}^{\prime}$ such that

$$
\mu_{(\omega, y)}^{s}\left(F_{k}^{\prime} \cap \eta_{\omega}^{s}(y)\right)=\mu_{(\omega, y)}^{s}\left(F_{k}^{\prime}\right)=\mu_{(\omega, y)}^{s}\left(F_{k}\right)>0 .
$$

Since $\mu_{(\omega, z)}^{s}=\mu_{(\omega, y)}^{s}$ for each $z \in F_{k}^{\prime} \cap \eta_{\omega}^{s}(y)$, it follows from Frostman's lemma (see [12, pp. 43]) that

$$
\operatorname{dim}_{H}\left(F_{k}^{\prime} \cap \eta_{\omega}^{s}(y)\right)=d^{s}
$$

Let us consider the collection of balls

$$
\mathcal{D}:=\left\{B\left(z, e^{-m_{j}(z)}\right): z \in F_{k}^{\prime} \cap \eta_{\omega}^{s}(y), j=1,2, \cdots\right\} .
$$

By the Besicovitch covering lemma (see, for example, [6] or [11]), one can find a countable subcover $\mathcal{D}^{\prime} \subset \mathcal{D}$ of $F_{k}^{\prime} \cap \eta_{\omega}^{s}(y)$ of arbitrarily small diameter and finite multiplicity $p$ ( $p$ depends only on the dimension of $M$ ). This means that for any $L \geq n_{1}$ one can choose a sequence of points $\left\{z_{i} \in F_{k}^{\prime} \cap \eta_{\omega}^{s}(y)\right\}_{i=1}^{\infty}$ and a sequence of integers $\left\{t_{i}\right\}_{i=1}^{\infty}$, where $t_{i} \in\left\{m_{j}\left(z_{i}\right)\right\}_{j=1}^{\infty}$ and $t_{i} \geq L$ for each $i$, such that the collection of balls

$$
\mathcal{D}^{\prime}=\left\{B\left(z_{i}, e^{-t_{i}}\right): i=1,2, \cdots\right\}
$$

comprises a cover of $F_{k}^{\prime} \cap \eta_{\omega}^{s}(y)$ whose multiplicity does not exceed $p$. We write $B_{i}=B\left(z_{i}, e^{-t_{i}}\right)$. The Hausdorff sum corresponding to this cover is

$$
S:=\sum_{B \in \mathcal{D}^{\prime}}(\operatorname{diam} B)^{d^{s}-\varepsilon}=2^{d^{s}-\varepsilon} \sum_{i=1}^{\infty} e^{-t_{i}\left(d^{s}-\varepsilon\right)} .
$$

By (5.3), noting that $a>1$, we obtain

$$
\begin{aligned}
\sum_{i=1}^{\infty} e^{-t_{i}\left(d^{s}-\varepsilon\right)} & \leq \sum_{i=1}^{\infty} \# \widehat{N}^{s}\left(t_{i}, B_{i} \cap \Gamma_{\omega}\right) \cdot 4 k C e^{-a t_{i}(h+2 \varepsilon)} \\
& \leq 4 k C \sum_{l=n_{1}}^{\infty} e^{-a l(h+2 \varepsilon)} \sum_{i: t_{i}=l} \# \widehat{N}^{s}\left(t_{i}, B_{i} \cap \Gamma_{\omega}\right) .
\end{aligned}
$$

Since the multiplicity of the subcover $\mathcal{D}^{\prime}$ is at most $p$, each set $B_{i}$ appears in the sum $\sum_{i: t_{i}=l} \# \widehat{N}^{s}\left(t_{i}, B_{i} \cap \Gamma_{\omega}\right)$ at most $p$ times. Furthermore, since $t_{i} \geq n_{1}$ and $z_{i} \in \alpha_{\omega}(x) \cap \widehat{\Gamma}_{\omega}$, by (4.22) we have

$$
B_{i} \cap \Gamma_{\omega}=B\left(z_{i}, e^{-t_{i}}\right) \cap \Gamma_{\omega} \subset \alpha_{\omega}\left(z_{i}\right)=\alpha_{\omega}(x) .
$$

Hence

$$
\sum_{i: t_{i}=l} \# \widehat{N}^{s}\left(t_{i}, B_{i} \cap \Gamma_{\omega}\right) \leq p \cdot \# \widehat{N}^{s}\left(l, \alpha_{\omega}(x)\right) .
$$


From Lemma 5.4 it follows that

$$
\begin{aligned}
S & \leq 4 k C 2^{d^{s}-\varepsilon} \sum_{l=n_{1}}^{\infty} e^{-a l(h+2 \varepsilon)} p \cdot \# \widehat{N}^{s}\left(l, \alpha_{\omega}(x)\right) \\
& \leq 4 k p C^{2} 2^{d^{s}-\varepsilon} \sum_{l=n_{1}}^{\infty} e^{-a l(h+2 \varepsilon)+a l(h+\varepsilon)} \\
& =4 k p C^{2} 2^{d^{s}-\varepsilon} \sum_{l=n_{1}}^{\infty} e^{-a l \varepsilon}<+\infty .
\end{aligned}
$$

Since $L$ can be chosen arbitrarily large (and so can the numbers $t_{i}$ ), it follows that

$$
\operatorname{dim}_{H}\left(F_{k}^{\prime} \cap \eta_{\omega}^{s}(y)\right) \leq d^{s}-\varepsilon<d^{s} .
$$

This contradicts (5.5). Hence $\mu_{\omega}\left(F_{k}\right)=0$ for any $k \geq 1$. This implies the first identity in the lemma. The other one can be proved in a similar way.

By Lemma 5.6, for $\mu$-a.e. $(\omega, x) \in \widehat{\Gamma}$ there exists an integer $n_{2}(\omega, x)$ (measurable in $(\omega, x))$ such that for $n \geq n_{2}(\omega, x)$ we have

$$
\begin{aligned}
& \# \widehat{N}^{s}\left(n, B\left(x, e^{-n}\right) \cap \Gamma_{\omega}\right)<\# N^{s}\left(n, x, B\left(x, e^{-n}\right)\right) e^{5 a n \varepsilon}, \\
& \# \widehat{N}^{u}\left(n, B\left(x, e^{-n}\right) \cap \Gamma_{\omega}\right)<\# N^{u}\left(n, x, B\left(x, e^{-n}\right)\right) e^{5 a n \varepsilon} .
\end{aligned}
$$

Moreover, by Lusin's theorem, there exists a subset $\Gamma^{\varepsilon} \subset \widehat{\Gamma}$ of measure $\mu\left(\Gamma^{\varepsilon}\right)>$ $\mu(\widehat{\Gamma})-\varepsilon>1-2 \varepsilon$ such that

$$
n_{\varepsilon}:=\sup \left\{n_{1}+2, n_{2}(\omega, x):(\omega, x) \in \Gamma^{\varepsilon}\right\}<\infty,
$$

and the inequalities (5.6) and (5.7) hold for every $n \geq n_{\varepsilon}$ and $(\omega, x) \in \Gamma^{\varepsilon}$. Furthermore, we can require that (4.30) (4.32) hold true for any $(\omega, x) \in \Gamma^{\varepsilon}$ and $n \geq n_{\varepsilon}$, with $\Gamma_{\omega}$ being replaced by $\widehat{\Gamma}_{\omega}$.

\section{Proof of Theorem 3.1}

Since Theorem 3.1(2) follows directly from Theorem [3.1(1) and (2.6), we will just prove Theorem 3.1(1). We will divide it into the following two lemmas.

Lemma 6.1. For each $(\omega, x) \in \Gamma^{\varepsilon}$ and $n \geq n_{\varepsilon}$,

$$
\mu_{(\omega, x)}^{s}\left(B^{s}\left(\omega, x ; e^{-n}\right)\right) \cdot \mu_{(\omega, x)}^{u}\left(B^{u}\left(\omega, x ; e^{-n}\right)\right) \leq \mu_{\omega}\left(B\left(x, 3 e^{-n}\right)\right) \cdot 2 C^{5} e^{7 a n \varepsilon} .
$$

Lemma 6.2. For each $(\omega, x) \in \Gamma^{\varepsilon}$ and $n \geq n_{\varepsilon}$,

$$
\mu_{\omega}\left(B\left(x, e^{-n}\right)\right) \leq \mu_{(\omega, x)}^{s}\left(B^{s}\left(\omega, x ; 4 e^{-n}\right)\right) \mu_{(\omega, x)}^{u}\left(B^{u}\left(\omega, x ; 4 e^{-n}\right)\right) \cdot 2 C^{3} e^{14 a n \varepsilon} .
$$

Proof of Lemma 6.1. Fix a point $(\omega, x) \in \Gamma^{\varepsilon}$ and an integer $n \geq n_{\varepsilon}$.

First we observe that

$$
B\left(z, e^{-n}\right) \subset B\left(x, 3 e^{-n}\right), \quad \forall z \in B\left(x, 2 e^{-n}\right) .
$$

Consider rectangles in $\mathcal{R}_{n}$ which intersect $B\left(x, 2 e^{-n}\right) \cap \Gamma_{\omega}$, and let $R$ be such a rectangle. Then there is a point $z \in B\left(x, 2 e^{-n}\right) \cap \Gamma_{\omega} \subset \alpha_{\omega}(x)$ such that $R=$ $\left[\alpha_{a n}^{a n}\right]_{\omega}(z)$. By (4.22) we have $R \subset B\left(z, e^{-n}\right)$ and hence $R \subset B\left(x, 3 e^{-n}\right)$. Therefore

$$
\begin{aligned}
\mu_{\omega}\left(B\left(x, 3 e^{-n}\right)\right) & \geq \sum_{R \in N\left(n, B\left(x, 2 e^{-n}\right) \cap \Gamma_{\omega}\right)} \mu_{\omega}(R) \\
& \geq \# N\left(n, B\left(x, 2 e^{-n}\right) \cap \Gamma_{\omega}\right) \cdot C^{-1} e^{-2 a n(h+\varepsilon)} .
\end{aligned}
$$


Then we observe that

$$
B\left(z, e^{-n}\right) \subset B\left(x, 2 e^{-n}\right), \quad \forall z \in B\left(x, e^{-n}\right) .
$$

Now let $R^{s}=\left[\alpha_{a n}^{0}\right]_{\omega}(z)$ be a rectangle in $\mathcal{F}_{n}^{s}$ which intersects $B\left(x, e^{-n}\right) \cap \widehat{\Gamma}_{\omega}$. We can assume $z \in B\left(x, e^{-n}\right) \cap \widehat{\Gamma}_{\omega}$. Then, for each rectangle

$$
R \in N^{u}\left(n, z,\left[\alpha_{a n}^{0}\right]_{\omega}(z) \cap B\left(z, e^{-n}\right)\right),
$$

$R$ intersects $B\left(z, e^{-n}\right) \cap \Gamma_{\omega}$ and hence also intersects $B\left(x, 2 e^{-n}\right) \cap \Gamma_{\omega}$ by (6.2). This means that $R$ is in fact a rectangle in $N\left(n, B\left(x, 2 e^{-n}\right) \cap \Gamma_{\omega}\right)$. For distinct rectangles $\left[\alpha_{a n}^{0}\right]_{\omega}(z)$ and $\left[\alpha_{a n}^{0}\right]_{\omega}\left(z^{\prime}\right)$ in $\widehat{N}^{s}\left(n, B\left(x, e^{-n}\right) \cap \Gamma_{\omega}\right)$, since $\left[\alpha_{a n}^{0}\right]_{\omega}(z) \cap$ $\left[\alpha_{a n}^{0}\right]_{\omega}\left(z^{\prime}\right)=\emptyset$, there is no common rectangle in both $N^{u}\left(n, z,\left[\alpha_{a n}^{0}\right]_{\omega}(z) \cap B\left(z, e^{-n}\right)\right)$ and $N^{u}\left(n, z^{\prime},\left[\alpha_{a n}^{0}\right]_{\omega}\left(z^{\prime}\right) \cap B\left(z^{\prime}, e^{-n}\right)\right)$. Thus

$$
\begin{aligned}
& \# N\left(n, B\left(x, 2 e^{-n}\right) \cap \Gamma_{\omega}\right) \\
& \geq \sum_{\left[\alpha_{a n}^{0}\right]_{\omega}(z) \in \widehat{N}^{s}}\left(n, B\left(x, e^{-n}\right) \cap \widehat{\Gamma}_{\omega}\right) \\
& \geq \# \widehat{N}^{s}\left(n, B\left(x, e^{-n}\right) \cap \widehat{\Gamma}_{\omega}\right) \\
& \quad \cdot \inf \left\{\# N^{u}\left(n, z,\left[\alpha_{a n}^{0}\right]_{\omega}(z) \cap B\left(z, e^{-n}\right)\right): z \in B\left(x, e^{-n}\right) \cap \widehat{\Gamma}_{\omega}\right\} .
\end{aligned}
$$

We now estimate $\# N^{u}\left(n, z,\left[\alpha_{a n}^{0}\right]_{\omega}(z) \cap B\left(z, e^{-n}\right)\right)$ for each $z \in \widehat{\Gamma}_{\omega}$. By (4.28) we have

$$
\eta_{\omega}^{u}(z) \cap\left[\alpha_{a n}^{0}\right]_{\omega}(z) \cap B^{u}\left(\omega, z ; e^{-n}\right) \subset \eta_{\omega}^{u}(z) \cap\left[\alpha_{a n}^{0}\right]_{\omega}(z) \cap B\left(z, e^{-n}\right) .
$$

Therefore by (4.34) and noting $a \geq 2$, one has

$$
\begin{aligned}
& \# N^{u}\left(n, z,\left[\alpha_{a n}^{0}\right]_{\omega}(z) \cap B\left(z, e^{-n}\right)\right) \\
& \quad \geq \frac{\mu_{(\omega, z)}^{u}\left(\left[\alpha_{a n}^{0}\right]_{\omega}(z) \cap B^{u}\left(\omega, z ; e^{-n}\right) \cap \Gamma_{\omega}\right)}{\max \left\{\mu_{(\omega, z)}^{u}(R): R \in N^{u}\left(n, z,\left[\alpha_{a n}^{0}\right]_{\omega}(z) \cap B\left(z, e^{-n}\right)\right)\right\}} \\
& \quad \geq e^{-n\left(d^{u}+\varepsilon\right)} \cdot C^{-1} e^{a n(h-\varepsilon)} \\
& \quad \geq C^{-1} e^{a n(h-2 \varepsilon)} \mu_{(\omega, x)}^{u}\left(B^{u}\left(\omega, x ; e^{-n}\right)\right) .
\end{aligned}
$$

Following the same line as in the proof of Lemma 5.5, we can prove that

$$
\# \widehat{N}^{s}\left(n, B\left(x, e^{-n}\right) \cap \widehat{\Gamma}_{\omega}\right) \geq \# N^{s}\left(n, x, B\left(x, e^{-n}\right) \cap \widehat{\Gamma}_{\omega}\right) \cdot C^{-2} e^{-2 a n \varepsilon} .
$$

Furthermore,

$$
\begin{aligned}
\# N^{s} & \left(n, x, B\left(x, e^{-n}\right) \cap \widehat{\Gamma}_{\omega}\right) \\
& \geq \frac{\mu_{(\omega, x)}^{s}\left(B\left(x, e^{-n}\right) \cap \widehat{\Gamma}_{\omega}\right)}{\max \left\{\mu_{(\omega, x)}^{s}(R): R \in N^{s}\left(n, x, B\left(x, e^{-n}\right) \cap \widehat{\Gamma}_{\omega}\right)\right\}} \\
& \geq \mu_{(\omega, x)}^{s}\left(B^{s}\left(\omega, x ; e^{-n}\right) \cap \widehat{\Gamma}_{\omega}\right) \cdot C^{-1} e^{a n(h-\varepsilon)} \\
& \geq \frac{1}{2 C} e^{a n(h-\varepsilon)} \cdot \mu_{(\omega, x)}^{s}\left(B^{s}\left(\omega, x ; e^{-n}\right)\right) .
\end{aligned}
$$

Putting (6.1)-(6.6) together, we obtain the inequality in the lemma.

Proof of Lemma 6.2, This lemma is a consequence of Lemmas [5.1, 5.2, 5.3 and inequalities (5.6) and (5.7). 


\section{THE CASE OF NONERGODIC MEASURES}

We show how to modify our arguments in the case when the measure $\mu$ is not ergodic. By an easy variant of Lemma 4.5 for nonergodic measures, there exists a $\Theta-$ invariant ( $\mu$-mod 0) function $h_{\mu}(\cdot): \Omega \times M \rightarrow \mathbf{R}^{+}$such that $h_{\mu}(F)=\int h_{\mu}(\omega, x) d \mu$ and (4.1)-(4.5) hold with $h_{\mu}(F)$ being replaced by $h_{\mu}(\omega, x)$. Then, by Lusin's theorem, given $\varepsilon^{\prime}>0$, there is a set $\Gamma^{\prime} \subset \Omega \times M$ with $\mu\left(\Gamma^{\prime}\right) \geq 1-\varepsilon^{\prime}$ such that $h_{\mu}(\omega, x), d_{\mu_{\omega}}^{s}(x), d_{\mu_{\omega}}^{u}(x)$, the smallest positive exponent $\lambda^{u}(\omega, x):=\lambda_{u(\omega, x)}(\omega, x)$ and the largest negative exponent $\lambda^{s}(\omega, x):=\lambda_{s(\omega, x)}(\omega, x)$ are all continuous on $\Gamma^{\prime}$. Let us fix $0<\delta<1$ and consider the sets

$$
\begin{aligned}
\Gamma(\omega, x):= & \left\{\left(\omega^{\prime}, x^{\prime}\right) \in \Omega \times M:\left|h_{\mu}(\omega, x)-h_{\mu}\left(\omega^{\prime}, x^{\prime}\right)\right|<\delta,\right. \\
& \left|d_{\mu_{\omega}}^{s}(x)-d_{\mu_{\omega^{\prime}}}^{s}\left(x^{\prime}\right)\right|<\delta,\left|d_{\mu_{\omega}}^{u}(x)-d_{\mu_{\omega^{\prime}}}^{u}\left(x^{\prime}\right)\right|<\delta, \\
& \left|\lambda^{u}(\omega, x)^{-1}-\lambda^{u}\left(\omega^{\prime}, x^{\prime}\right)^{-1}\right|<\delta, \\
& \left.\left|\lambda^{s}(\omega, x)^{-1}-\lambda^{s}\left(\omega^{\prime}, x^{\prime}\right)^{-1}\right|<\delta\right\}
\end{aligned}
$$

which are clearly $\Theta$-invariant ( $\mu$-mod 0$)$. These sets, restricted to $\Gamma^{\prime}$, form an open cover of $\Gamma^{\prime}$ by our requirement. Since $\Omega \times M$ is a Polish space, it is Lindelöf, and hence so is its subset $\Gamma^{\prime}$. Therefore there exists a countable sub-collection of sets $\left\{\Gamma^{i}:=\Gamma\left(\omega^{i}, x^{i}\right)\right\}_{i \geq 1}\left(\right.$ with $\mu\left(\Gamma^{i}\right)>0$ for each $i$ ) which still covers $\Gamma^{\prime}$. Let $\mu^{i}$ be the conditional measure of $\mu$ on $\Gamma^{i}$, and it is obviously $\Theta$-invariant. Since $\mu$ satisfies (2.1), so does $\mu^{i}$. Therefore the results presented in Section 2 can be applied to $\mu^{i}$, yielding the existence of the local stable and unstable dimension, $d_{\mu_{\omega}^{i}}^{s}(x)$ and $d_{\mu_{\omega}^{i}}^{u}(x)$, of $\mu^{i}$. Since for $\mu$-a.e. $(\omega, x) \in \Gamma^{i}$

$$
h_{\mu^{i}}(\omega, x)=h_{\mu}(\omega, x), \quad d_{\mu_{\omega}^{i}}^{s}(x)=d_{\mu_{\omega}}^{s}(x), \quad d_{\mu_{\omega}^{i}}^{u}(x)=d_{\mu_{\omega}}^{u}(x),
$$

the following inequalities hold for $\mu$-a.e. $(\omega, x) \in \Gamma^{i}$ :

$$
\left|h_{\mu^{i}}(\omega, x)-h_{i}\right|<\delta, \quad\left|d_{\mu_{\omega}^{i}}^{s}(x)-d_{i}^{s}\right|<\delta, \quad\left|d_{\mu_{\omega}^{i}}^{u}(x)-d_{i}^{u}\right|<\delta,
$$

where $h_{i}:=h_{\mu}\left(\omega^{i}, x^{i}\right), d_{i}^{s}:=d_{\mu_{\omega^{i}}}^{s}\left(x^{i}\right)$ and $d_{i}^{u}:=d_{\mu_{\omega^{i}}}^{u}\left(x^{i}\right)$. Then we can apply the arguments in the proof of Theorem 3.1 to the measure $\mu^{i}$, showing that for $\mu$-a.e. $(\omega, x) \in \Gamma^{i}$ the following inequalities hold true:

$$
\begin{aligned}
& \underline{d}_{\mu_{\omega}^{i}}(x) \geq d_{\mu_{\omega}^{i}}^{s}(x)+d_{\mu_{\omega}^{i}}^{u}(x)-c_{i} \delta, \\
& \bar{d}_{\mu_{\omega}^{i}}(x) \leq d_{\mu_{\omega}^{i}}^{s}(x)+d_{\mu_{\omega}^{i}}^{u}(x)+c_{i} \delta,
\end{aligned}
$$

where $\underline{d}_{\mu_{\omega}^{i}}(x)$ and $\bar{d}_{\mu_{\omega}^{i}}(x)$ are the lower and upper pointwise dimension of $\mu_{\omega}^{i}$, and $c_{i}$ is a constant which is independent of $(\omega, x)$ or $\delta$. In fact, one can take $c_{i}=12 a_{i}$, where $a_{i}$ is the integral part of $4+2 \max \left\{\lambda^{u}\left(\omega^{i}, x^{i}\right)^{-1},\left|\lambda^{s}\left(\omega^{i}, x^{i}\right)\right|^{-1}\right\}$. To see this, one needs to go through slight modifications of the arguments in Section 4 and the proofs of Lemmas 5.1 5.6, 6.1 and 6.2. made by replacing $\mu, a, h, d^{s}$ and $d^{u}$ respectively with $\mu^{i}, a_{i}, h_{i}, d_{i}^{s}$ and $d_{i}^{u}$ and taking $\delta$ into account due to (7.2). For example, the first inequality of Lemma 5.1 now reads

$$
\# N^{s}\left(n, x, B\left(x, e^{-n}\right)\right) \leq\left(\mu^{i}\right)_{(\omega, x)}^{s}\left(B^{s}\left(\omega, x ; 4 e^{-n}\right)\right) \cdot C e^{a_{i} n\left(h_{i}+\delta+\varepsilon\right)} ;
$$

to modify Lemma 5.6, replace (5.4) with the inequality

$$
\lim _{\rho \rightarrow 0} \frac{\log \left(\mu^{i}\right)_{(\omega, z)}^{s}\left(B^{s}(\omega, z ; \rho)\right)}{\log \rho}=d_{\mu_{\omega}^{i}}^{s}(z) \geq d_{i}^{s}-\delta
$$


and apply the nonuniform mass distribution principle (see [12, pp. 43]) instead of Frostman's lemma, yielding

$$
\operatorname{dim}_{H}\left(F_{k}^{\prime} \cap \eta_{\omega}^{s}(y)\right) \geq d_{i}^{s}-\delta
$$

which takes the place of (5.5). Then the limits in Lemma 5.6 can be modified to

$$
\begin{gathered}
\varlimsup_{n \rightarrow+\infty} \frac{\# \widehat{N}^{s}\left(n, B\left(y, e^{-n}\right) \cap \Gamma_{\omega}\right)}{\# N^{s}\left(n, y, B\left(y, e^{-n}\right)\right)} e^{-a_{i} n(5 \varepsilon+4 \delta)}=0, \\
\varlimsup_{n \rightarrow+\infty} \frac{\# \widehat{N}^{u}\left(n, B\left(y, e^{-n}\right) \cap \Gamma_{\omega}\right)}{\# N^{u}\left(n, y, B\left(y, e^{-n}\right)\right)} e^{-a_{i} n(5 \varepsilon+4 \delta)}=0 .
\end{gathered}
$$

From these it can be seen that one may take $c_{i}=12 a_{i}$.

It follows from Proposition 4.7 that for $\mu$-a.e. $(\omega, x) \in \Gamma^{i}$

$$
\underline{d}_{\mu_{\omega}^{i}}(x)=\underline{d}_{\mu_{\omega}}(x), \quad \bar{d}_{\mu_{\omega}^{i}}(x)=\bar{d}_{\mu_{\omega}}(x) .
$$

Noting that for $\mu$-a.e. $(\omega, x) \in \Gamma^{i}$

$$
\left|\lambda^{u}\left(\omega^{i}, x^{i}\right)\right|^{-1}<\left|\lambda^{u}(\omega, x)\right|^{-1}+1, \quad\left|\lambda^{s}\left(\omega^{i}, x^{i}\right)\right|^{-1}<\left|\lambda^{s}(\omega, x)\right|^{-1}+1,
$$

by (7.1), (7.3) and (7.4), we have the following inequalities for $\mu$-a.e. $(\omega, x) \in \Gamma^{i}$ and each $i \geq 1$ :

$$
\begin{aligned}
& \underline{d}_{\mu_{\omega}}(x) \geq d_{\mu_{\omega}}^{s}(x)+d_{\mu_{\omega}}^{u}(x)-c \delta, \\
& \bar{d}_{\mu_{\omega}}(x) \leq d_{\mu_{\omega}}^{s}(x)+d_{\mu_{\omega}}^{u}(x)+c \delta,
\end{aligned}
$$

where $c=c(\omega, x):=24 \max \left\{\left|\lambda^{u}(\omega, x)\right|^{-1}+3,\left|\lambda^{s}(\omega, x)\right|^{-1}+3\right\}$. Since $\left\{\Gamma^{i}\right\}_{i \geq 1}$ covers $\Gamma^{\prime}$, (7.5) and (7.6) hold true for $\mu$-a.e. $(\omega, x) \in \Gamma^{\prime}$. Letting $\delta \rightarrow 0$, we know that for $\mu$-a.e. $(\omega, x) \in \Gamma^{\prime}$

$$
\underline{d}_{\mu_{\omega}}(x)=\bar{d}_{\mu_{\omega}}(x)=d_{\mu_{\omega}}^{s}(x)+d_{\mu_{\omega}}^{u}(x) .
$$

Since $\varepsilon^{\prime}>0$ is arbitrary and $\mu\left(\Gamma^{\prime}\right) \geq 1-\varepsilon^{\prime}$, (7.7) holds for $\mu$-a.e. $(\omega, x) \in \Omega \times M$. This proves the last statement of Theorem 3.1.

\section{Proofs of (4.33) AND (4.34)}

In this section we give proofs of requirements (4.33) and (4.34). We will inherit many notations introduced in [13 without announcing beforehand. In fact we will prove

Proposition 8.1. Let $\mu$ be ergodic and let $\alpha, \eta^{s}, \eta^{u}$ and $\Gamma$ be as introduced in Section 4. Then for $\mu$-a.e. $(\omega, x) \in \Gamma$ one has

$$
\begin{aligned}
& \lim _{n \rightarrow+\infty}-\frac{1}{n} \log \mu_{(\omega, x)}^{s}\left(\left[\alpha_{0}^{a n}\right]_{\omega}(x) \cap B^{s}\left(\omega, x ; e^{-n}\right) \cap \Gamma_{\omega}\right)=d^{s}, \\
& \lim _{n \rightarrow+\infty}-\frac{1}{n} \log \mu_{(\omega, x)}^{u}\left(\left[\alpha_{a n}^{0}\right]_{\omega}(x) \cap B^{u}\left(\omega, x ; e^{-n}\right) \cap \Gamma_{\omega}\right)=d^{u} .
\end{aligned}
$$

We will only prove equation (8.2) since the other one can be obtained by applying the same arguments to the system $\Theta^{-1}: \Omega \times M \hookleftarrow$. The proof goes essentially along the same line as that of [13]. 


\subsection{Pseudo-pointwise dimensions and pseudo-local entropies. Let}

$$
\widetilde{\alpha}:=\alpha \vee\{\Gamma, \Omega \times M \backslash \Gamma\} .
$$

Clearly we have $H_{\mu}(\widetilde{\alpha} \mid \sigma) \leq H_{\mu}(\alpha \mid \sigma)+\log 2<+\infty$. In view of (4.7) and (2.6) it is an easy exercise to check that (8.2) follows directly from the proposition below.

Proposition 8.2. Let $\eta^{u}$ and $\widetilde{\alpha}$ be as introduced above. Then for $\mu$-a.e. $(\omega, x)$

$$
\lim _{n \rightarrow+\infty}-\frac{1}{n} \log \mu_{(\omega, x)}^{\eta^{u} \vee \widetilde{\alpha}_{a n}^{0}}\left(B^{u}\left(\omega, x ; e^{-n}\right)\right)=d^{u} .
$$

The limit in the above proposition looks very much like the pointwise dimension of $\mu$ on the $W^{u}$-manifold at $(\omega, x)$ defined in Section 2 and, for the sake of language, in this section we name it as the pseudo-pointwise dimension of $\mu$ on the $W^{u_{-}}$ manifold at $(\omega, x)$. The proposition states that the pseudo-pointwise dimension coincides with the usual pointwise dimension.

We will prove the existence of pseudo-pointwise dimension on $W^{u}$-manifolds and its coincidence with $d^{u}$ through quantities named pseudo-pointwise dimensions on $W^{i}$-manifolds and pseudo-local entropies along $W^{i}$-manifolds $(i=1,2, \cdots, u)$, which we will introduce below.

We begin with the following notations. Let $\eta^{1}>\eta^{2}>\cdots>\eta^{u}$ be a sequence of increasing measurable partitions of $\Omega \times M$ with each $\eta^{i}$ being subordinate to the corresponding $W^{i}$-manifolds. Let $n:(0,1) \rightarrow \mathbf{Z}^{+}$and $b: \mathbf{Z}^{+} \rightarrow \mathbf{Z}^{+}$be two monotonic functions such that

$$
0<\lim _{\rho \downarrow 0}-\frac{n(\rho)}{\log \rho}<+\infty, \quad 0<\lim _{n \rightarrow+\infty} \frac{b(n)}{n}<+\infty
$$

(such functions will be called well-rated functions in this section; in the subsequent arguments one may take $n(\rho)=[-a \log \rho]$ and $b(n)=[b n]$ for some positive numbers $a$ and $b$, where $[t]$ denotes the integral part of a real number $t)$. Put

$$
\begin{aligned}
\underline{\delta}_{i}^{\prime}(\omega, x) & :=\varliminf_{\rho \rightarrow 0} \log \mu_{(\omega, x)}^{\eta^{i} \vee \widetilde{\alpha}_{n(\rho)}^{0}}\left(B^{i}(\omega, x ; \rho)\right) / \log \rho, \\
\bar{\delta}_{i}^{\prime}(\omega, x) & :=\varlimsup_{\rho \rightarrow 0} \log \mu_{(\omega, x)}^{\eta^{i} \vee \widetilde{\alpha}_{n(\rho)}^{0}}\left(B^{i}(\omega, x ; \rho)\right) / \log \rho, \\
\underline{h}_{i}^{\prime}(\omega, x ; \rho) & :=\varliminf_{n \rightarrow+\infty}-\frac{1}{n} \log \mu_{(\omega, x)}^{\eta^{i} \vee \widetilde{\alpha}_{b(n)}^{0}}\left(B^{i}(\omega, x ; n, \rho)\right), \\
\bar{h}_{i}^{\prime}(\omega, x ; \rho) & :=\varlimsup_{n \rightarrow+\infty}-\frac{1}{n} \log \mu_{(\omega, x)}^{\eta^{i} \vee \widetilde{\alpha}_{b(n)}^{0}}\left(B^{i}(\omega, x ; n, \rho)\right), \\
\underline{h}_{i}^{\prime}(\omega, x) & :=\lim _{\rho \downarrow 0} \underline{h}_{i}^{\prime}(\omega, x ; \rho), \\
\bar{h}_{i}^{\prime}(\omega, x) & :=\lim _{\rho \downarrow 0} \bar{h}_{i}^{\prime}(\omega, x ; \rho),
\end{aligned}
$$

where $B^{i}(\omega, x ; \rho)$ and $B^{i}(\omega, x ; n, \rho)$ are as defined in [13], i.e.,

$$
\begin{aligned}
B^{i}(\omega, x ; \rho) & :=\left\{y \in W^{i}(\omega, x): d_{(\omega, x)}^{i}(x, y)<\rho\right\}, \\
B^{i}(\omega, x ; n, \rho) & :=\left\{y \in W^{i}(\omega, x): d_{\Theta^{k}(\omega, x)}^{i}\left(f_{\omega}^{k} x, f_{\omega}^{k} y\right)<\rho \text { for } 0 \leq k \leq n\right\} .
\end{aligned}
$$

We call $\underline{\delta}_{i}^{\prime}(\omega, x)$ and $\bar{\delta}_{i}^{\prime}(\omega, x)$, respectively, the lower and the upper pseudo-pointwise dimension of $\mu$ on the $W^{i}$-manifold at $(\omega, x)$, and $\underline{h}_{i}^{\prime}(\omega, x), \bar{h}_{i}^{\prime}(\omega, x)$ the lower and the upper pseudo-local entropy along $W^{i}$-manifolds at $(\omega, x)$. 
Sometimes we write $\underline{\delta}_{i}^{\prime}(\omega, x ; \widetilde{\alpha}):=\underline{\delta}_{i}^{\prime}(\omega, x)$ to indicate the dependence of this quantity on the partition $\widetilde{\alpha}$. Other similar notations have analogous meanings.

Remark 8.3. By [4.7] and from [13] one can easily check

$$
\begin{aligned}
\bar{\delta}_{i}^{\prime}(\omega, x) \geq \underline{\delta}_{i}^{\prime}(\omega, x) & \geq \delta_{i} \\
\bar{h}_{i}^{\prime}(\omega, x ; \rho) \geq \underline{h}_{i}^{\prime}(\omega, x ; \rho) & \geq \underline{h}_{i}(\omega, x ; \rho)
\end{aligned}
$$

and hence

$$
\bar{h}_{i}^{\prime}(\omega, x) \geq \underline{h}_{i}^{\prime}(\omega, x) \geq h_{i}
$$

for $\mu$-a.e. $(\omega, x)$ and each $i \leq u$, where $\delta_{i}, \underline{h}_{i}$ and $h_{i}$ are introduced in [13].

It would be clear later that the quantities $\underline{\delta}_{i}^{\prime}, \bar{\delta}_{i}^{\prime}, \underline{h}_{i}^{\prime}$ and $\bar{h}_{i}^{\prime}$ are in fact independent of the choices of the well-rated functions $n(\cdot)$ and $b(\cdot)$.

We point out that for $\mu$-a.e. $(\omega, x)$ and $i=1,2, \cdots, u$

$$
\left[\eta^{i} \vee \alpha_{0}^{n}\right]_{\omega}(x) \subset B^{i}\left(\omega, x ; n, \delta_{*}\right), \quad \forall n \geq n_{0}(\omega, x)
$$

and, for each $y \in\left[\eta^{i} \vee \alpha_{0}^{n}\right]_{\omega}(x)$ with $n \geq n_{0}(\omega, x), f_{\omega}^{k} y$ stays well inside the $\left(\varepsilon_{*}, \ell_{*}\right)$ chart at $\Theta^{k}(\omega, x)$ for $k=0,1, \cdots, n$. For $i=u$, this has already been stated in Lemma 4.4. for a general $i$, the conclusion can be proved analogously, and the reader is referred to [13] for details. These facts will be our starting point of many other basic estimates.

8.2. Coincidence of pseudo and usual local entropies. Let $\widetilde{\alpha}$ be the ( fixed) partition introduced in the last subsection. For each sufficiently small $\varepsilon>0$, in particular, $\varepsilon<\varepsilon_{*}$, we choose a system of $(\varepsilon, \ell)$-Lyapunov charts $\left\{\Phi_{(\omega, x)}\right\}$ given by [13, Prop. 3.1].

First observe that $\ell_{*}(\omega, x) \leq \ell(\omega, x)$ and hence

$$
\ell_{*}\left(\Theta^{n}(\omega, x)\right) \leq \ell(\omega, x) e^{|n| \varepsilon}, \quad \forall n \in \mathbf{Z},
$$

for $\mu$-a.e. $(\omega, x)$. This can be deduced directly from the explicit formula of $\ell(\omega, x)$ (see, for example, [11, pp. 142-145]). The above observation helps us switching from one system of Lyapunov charts with a fixed parameter $\varepsilon_{*}$ to another system of Lyapunov charts with an arbitrarily smaller parameter $\varepsilon$.

Then we will prove that $\bar{h}_{i}^{\prime}(\omega, x) \leq h_{i}$ for any $i \leq u$, and hence the pseudo-local entropies coincide with the usual local entropies. Before doing this, we first prove the following fact.

Lemma 8.4. Let $\lambda:=\min \left\{\left|\lambda_{i}\right|: i=1,2, \cdots, r\right\}$ and let $p \in(0,1)$ be given. Let $\beta:=\widetilde{\alpha}_{0}^{m}$ for some $m \in \mathbf{Z}^{+}$. Then for $\mu$-a.e. $(\omega, x)$ we have

$$
\left[\eta^{i} \vee \beta_{0}^{n}\right]_{\omega}(x) \subset B^{i}\left(\omega, x ;[n p], e^{-m\left(\lambda_{i}-3 \varepsilon_{*}\right)}\right), \quad \forall n \geq n_{0}^{\prime}(\omega, x),
$$

where $n_{0}^{\prime}: \Omega \times M \rightarrow \mathbf{Z}^{+}$is a Borel function independent of $m$.

Proof. In fact, in the proof of [13, Prop. 5.1] we have already proved

$$
d_{\Theta^{k}(\omega, x)}^{i}\left(f_{\omega}^{k} x, f_{\omega}^{k} y\right) \leq 2 K_{*} \delta_{*} \ell_{*}\left(\Theta^{k}(\omega, x)\right) e^{-(n-k)\left(\lambda_{i}-3 \varepsilon_{*}\right)}, \quad k=0,1, \cdots, n,
$$

for any $y \in\left[\eta^{i} \vee \alpha_{0}^{n}\right]_{\omega}(x)$ with $n \geq n_{0}(\omega, x)$ and for $\mu$-a.e. $(\omega, x)$. Let

$$
\varepsilon_{0}:=\min \left\{\frac{\varepsilon_{*}}{2}, \frac{1-p}{3}\left(\lambda-3 \varepsilon_{*}\right)\right\},
$$


and take a system of $\left(\varepsilon_{0}, \ell_{0}\right)$-Lyapunov charts (where $\ell_{0}(\omega, x):=\ell(\omega, x)$ is a function introduced in [13, Prop. 3.1] corresponding to the parameter $\left.\varepsilon=\varepsilon_{0}\right)$. Then, by (8.10), for $k=0,1, \cdots, n$ we have

$$
d_{\Theta^{k}(\omega, x)}^{i}\left(f_{\omega}^{k} x, f_{\omega}^{k} y\right) \leq 2 K_{*} \delta_{*} \ell_{0}(\omega, x) e^{-(n-k)\left(\lambda_{i}-3 \varepsilon_{*}\right)+k \varepsilon_{0}} .
$$

Fix an integer $m \geq 0$. In view of the facts $\widetilde{\alpha} \geq \alpha,(1-p)\left(\lambda_{i}-3 \varepsilon_{*}\right) \geq 3 \varepsilon_{0}$ and (8.12), for any $y \in\left[\eta^{i} \vee \widetilde{\alpha}_{0}^{n+m}\right]_{\omega}(x)$ with $n \geq n_{0}(\omega, x)$ we have

$$
d_{\Theta^{k}(\omega, x)}^{i}\left(f_{\omega}^{k} x, f_{\omega}^{k} y\right) \leq 2 K_{*} \delta_{*} \ell_{0}(\omega, x) e^{-m\left(\lambda_{i}-3 \varepsilon_{*}\right)-2 n \varepsilon_{0}}, \quad k=0,1, \cdots,[n p] .
$$

This implies

$$
\left[\eta^{i} \vee \widetilde{\alpha}_{0}^{n+m}\right] \subset B^{i}\left(\omega, x ;[n p], 2 K_{*} \delta_{*} \ell_{0}(\omega, x) e^{-m\left(\lambda_{i}-3 \varepsilon_{*}\right)-2 n \varepsilon_{0}}\right)
$$

for $\mu$-a.e. $(\omega, x)$ and any $n \geq n_{0}(\omega, x)$. The conclusion (8.11) then becomes obvious with $n_{0}^{\prime}(\omega, x)$ being defined by

$$
n_{0}^{\prime}(\omega, x):=\max \left\{n_{0}(\omega, x), 1+\left[\frac{1}{2 \varepsilon_{0}} \log \left(2 K_{*} \delta_{*} \ell_{0}(\omega, x)\right)\right]\right\} .
$$

Lemma 8.5. If $\beta=\widetilde{\alpha}_{0}^{m}$ for some $m \in \mathbf{Z}^{+}$, then

$$
\lim _{n \rightarrow+\infty}-\frac{1}{n} \log \mu_{(\omega, x)}^{\eta^{i} \vee \widetilde{\alpha}_{b(n)}^{0}}\left(\left[\left(\eta^{i} \vee \beta\right)_{0}^{n}\right]_{\omega}(x)\right)=H_{\mu}\left(\eta^{i} \mid \Theta \eta^{i} \vee \sigma\right)=h_{i}
$$

holds for $\mu$-a.e. $(\omega, x)$, where $b: \mathbf{Z}^{+} \rightarrow \mathbf{Z}^{+}$is a well-rated function.

Proof. Since $\beta=\widetilde{\alpha}_{0}^{m}$, for any $n$ large enough we can write

$$
\begin{aligned}
& -\log \mu_{(\omega, x)}^{\eta^{i} \vee \widetilde{\alpha}_{b(n)}^{0}}\left(\left[\left(\eta^{i} \vee \beta\right)_{0}^{n}\right]_{\omega}(x)\right) \\
= & I_{\mu}\left(\left[\eta^{i} \vee \beta\right]_{0}^{n} \mid \eta^{i} \vee \widetilde{\alpha}_{b(n)}^{0} \vee \sigma\right)(\omega, x) \\
= & I_{\mu}\left(\beta \mid \eta^{i} \vee \widetilde{\alpha}_{b(n)}^{0} \vee \sigma\right)(\omega, x) \\
& +\sum_{k=1}^{n} I_{\mu}\left(\beta \vee \eta^{i} \mid \Theta \eta^{i} \vee \Theta \beta_{k-1}^{0} \vee \Theta^{k} \widetilde{\alpha}_{b(n)}^{0} \vee \sigma\right)\left(\Theta^{k}(\omega, x)\right) \\
= & I_{\mu}\left(\beta \mid \eta^{i} \vee \widetilde{\alpha}_{b(n)}^{0} \vee \sigma\right)(\omega, x) \\
& +\sum_{k=1}^{n} I_{\mu}\left(\beta \vee \eta^{i} \mid \Theta \eta^{i} \vee \Theta \widetilde{\alpha}_{b(n)+k-1}^{m} \vee \sigma\right)\left(\Theta^{k}(\omega, x)\right) \\
= & I_{\mu}\left(\beta \mid \eta^{i} \vee \widetilde{\alpha}_{b(n)}^{0} \vee \sigma\right)(\omega, x) \\
& +\sum_{k=1}^{n} I_{\mu}\left(\beta \vee \eta^{i} \mid \Theta \eta^{i} \vee \Theta \beta_{b(n)+k-1}^{0} \vee \sigma\right)\left(\Theta^{k}(\omega, x)\right) .
\end{aligned}
$$

Clearly we have

$$
I_{n}(\omega, x):=I_{\mu}\left(\beta \vee \eta^{i} \mid \Theta \eta^{i} \vee \Theta \beta_{n}^{0} \vee \sigma\right)(\omega, x) \rightarrow I_{\infty}(\omega, x)
$$

in $L^{1}$ and $\mu$-a.e. as $n$ tends to $+\infty$ with $I_{\infty}(\omega, x):=I_{\mu}\left(\beta \vee \eta^{i} \mid \Theta \eta^{i} \vee \Theta \beta^{-} \vee \sigma\right)(\omega, x)$. Note also that

$$
H_{\mu}(\beta \mid \sigma) \leq(m+1) H_{\mu}(\widetilde{\alpha} \mid \sigma)<+\infty
$$

and

$$
\int I_{\infty}(\omega, x) d \mu=H_{\mu}\left(\beta \vee \eta^{i} \mid \Theta \eta^{i} \vee \Theta \beta^{-} \vee \sigma\right)=H_{\mu}\left(\eta^{i} \mid \Theta \eta^{i} \vee \sigma\right)=h_{i}
$$


which can be proved following the idea of [11, Lemma VI.5.4]. Then a slight variant of the Birkhoff ergodic theorem gives

$$
\varlimsup_{n \rightarrow+\infty}-\frac{1}{n} \log \mu_{(\omega, x)}^{\eta^{i} \vee \widetilde{\alpha}_{b(n)}^{0}}\left(\left[\left(\eta^{i} \vee \beta\right)_{0}^{n}\right]_{\omega}(x)\right)=\int I_{\infty}(\omega, x) d \mu=h_{i}
$$

for $\mu$-a.e. $(\omega, x)$.

By means of the above lemmas, we can now prove the following proposition which tells the coincidence of the pseudo-local entropies with the usual local entropies.

Proposition 8.6. $\underline{h}_{i}^{\prime}(\omega, x)=\bar{h}_{i}^{\prime}(\omega, x)=H_{\mu}\left(\eta^{i} \mid \Theta \eta^{i} \vee \sigma\right)=h_{i}$ for $\mu$-a.e. $(\omega, x)$ and $1 \leq i \leq u$, where $h_{i}$ is the local entropy along $W^{i}$-manifolds defined in [13, Sect. 4].

Proof. Due to Remark 8.3 , one needs only to prove

$$
\bar{h}_{i}^{\prime}(\omega, x) \leq h_{i} .
$$

Given $\delta>0$ arbitrarily, let $m \geq 1$ be an integer so that $e^{-m\left(\lambda-3 \varepsilon_{*}\right)} \leq \delta$. Put $\beta:=\widetilde{\alpha}_{0}^{m}$. Then, for any $p \in(0,1)$, by Lemma 8.4 we have for $\mu$-a.e. $(\omega, x)$

$$
\left[\left(\eta^{i} \vee \beta\right)_{0}^{n}\right]_{\omega}(x) \subset B^{i}(\omega, x ;[n p], \delta) \quad \text { for any } n \geq n_{0}^{\prime}(\omega, x) .
$$

Since $p \in(0,1)$, we have $\left\{[n p]: n \in \mathbf{Z}^{+}\right\}=\mathbf{Z}^{+}$and hence

$$
\begin{aligned}
\bar{h}_{i}^{\prime}(\omega, x ; \delta) & =\varlimsup_{n \rightarrow+\infty}-\frac{1}{n} \log \mu_{(\omega, x)}^{\eta^{i} \vee \widetilde{\alpha}_{b(n)}^{0}}\left(B^{i}(\omega, x ; n, \delta)\right) \\
& =\varlimsup_{n \rightarrow+\infty}-\frac{1}{[n p]} \log \mu_{(\omega, x)}^{\eta^{i} \vee \widetilde{\alpha}_{b([n p])}^{0}}\left(B^{i}(\omega, x ;[n p], \delta)\right) \\
& \leq \frac{1}{p} \cdot \varlimsup_{n \rightarrow+\infty}-\frac{1}{n} \log \mu_{(\omega, x)}^{\eta^{i} \vee \widetilde{\alpha}_{b([n p])}^{0}}\left(\left[\left(\eta^{i} \vee \beta\right)_{0}^{n}\right]_{\omega}(x)\right),
\end{aligned}
$$

where $b(\cdot)$ is a well-rated function. Clearly $b^{\prime}(n):=b([n p])$ is still well-rated for any fixed $p \in(0,1)$. Thus, by Lemma 8.5. the limit in (8.13) is $h_{i}$ for any fixed $p>0$. Hence we have proved

$$
\bar{h}_{i}^{\prime}(\omega, x ; \delta) \leq h_{i} / p
$$

for any $\delta>0$ and any $p \in(0,1)$. This proves $\bar{h}_{i}^{\prime}(\omega, x) \leq h_{i}$. $\widetilde{\alpha}$.

As needed in the next subsection, we deduce another property of the partition

Lemma 8.7. Let $m \geq 1, \beta:=\widetilde{\alpha}_{0}^{m}$ and $\varepsilon \in\left(0, \varepsilon_{*}\right)$ be given. Then there exists a Borel function $n_{0}^{\prime \prime}: \Omega \times M \rightarrow \mathbf{Z}^{+}$(independent of $m$ ) such that for $\mu$-a.e. $(\omega, x)$

$$
\left[\eta^{i} \vee \beta_{0}^{n}\right]_{\omega}(x) \subset B^{i}\left(\omega, x ; e^{-n\left(\lambda_{i}-9 \varepsilon\right)}\right), \quad \forall n \geq n_{0}^{\prime \prime}(\omega, x) .
$$

Furthermore, for any well-rated function $b(\cdot)$ and $\mu$-a.e. $(\omega, x)$ we have

$$
\lim _{n \rightarrow+\infty}-\frac{1}{n} \log \mu_{(\omega, x)}^{\eta^{i} \vee \widetilde{\alpha}_{b(n)}^{0}}\left(\left[\beta_{0}^{n}\right]_{\omega}(x)\right)=h_{i}
$$

Proof. From the proof of Lemma 8.4 we have

$$
d_{\Theta^{k}(\omega, x)}^{i}\left(f_{\omega}^{k} x, f_{\omega}^{k} y\right) \leq 2 K_{*} \delta_{*} \ell_{*}\left(\Theta^{k}(\omega, x)\right) e^{-(n+m-k)\left(\lambda_{i}-3 \varepsilon_{*}\right)}
$$

for any $y \in\left[\eta^{i} \vee \beta_{0}^{n}\right]_{\omega}(x)$ and $k=0,1, \cdots, n+m$ with $n \geq n_{0}(\omega, x)$. By (8.10) one has

$$
d_{\Theta^{k}(\omega, x)}^{i}\left(f_{\omega}^{k} x, f_{\omega}^{k} y\right) \leq 2 K_{*} \delta_{*} \ell(\omega, x) e^{-(n+m-k)\left(\lambda_{i}-3 \varepsilon_{*}\right)+k \varepsilon}
$$


for $k=0,1, \cdots, n+m$ since $\varepsilon<\varepsilon_{*}$. Then, noting that $\lambda_{i} \geq 200 \varepsilon_{*}$, we have $p:=1-\frac{4 \varepsilon}{\lambda_{i}-3 \varepsilon_{*}} \in(0,1)$. Hence, for any

$$
n \geq n^{\prime}(\omega, x):=\max \left\{n_{0}(\omega, x), 4+\left[\frac{1}{\varepsilon} \log \left(2 K_{0} K_{*} \delta_{*} \ell(\omega, x)^{3}\right)\right]\right\}
$$

(where $K_{0}$ is a universal constant given by [13, Prop. 3.1]), we have

$$
d_{\Theta^{k}(\omega, x)}^{i}\left(f_{\omega}^{k} x, f_{\omega}^{k} y\right) \leq K_{0}^{-1} \ell\left(\Theta^{k}(\omega, x)\right)^{-2}, \quad k=0,1, \cdots,[n p]+1,
$$

for any $y \in\left[\eta^{i} \vee \beta_{0}^{n}\right]_{\omega}(x)$. This means that $f_{\omega}^{k} y, k=0,1, \cdots,[n p]+1$, stay well inside the $(\varepsilon, \ell)$-charts. Therefore we can employ $(\varepsilon, \ell)$-charts for these points instead of $\left(\varepsilon_{*}, \ell_{*}\right)$-charts, yielding that (noting $p=1-\frac{4 \varepsilon}{\lambda_{i}-3 \varepsilon_{*}}$ and $\lambda_{i} \geq 200 \varepsilon_{*}$ )

$$
\begin{aligned}
d_{(\omega, x)}^{i}(x, y) & \leq 2 K_{0} \delta_{*} \ell(\omega, x) e^{-([n p]+1)\left(\lambda_{i}-3 \varepsilon\right)} \\
& \leq 2 K_{0} \delta_{*} \ell(\omega, x) e^{-n p\left(\lambda_{i}-3 \varepsilon\right)} \\
& \leq 2 K_{0} \delta_{*} \ell(\omega, x) e^{-n\left(\lambda_{i}-9 \varepsilon+\frac{2 \lambda_{i}-18 \varepsilon_{*}}{\lambda_{i}-3 \varepsilon_{*}} \varepsilon\right)} \\
& \leq 2 K_{0} \delta_{*} \ell(\omega, x) e^{-n \varepsilon} \cdot e^{-n\left(\lambda_{i}-9 \varepsilon\right)}
\end{aligned}
$$

for any $y \in\left[\eta^{i} \vee \beta_{0}^{n}\right]_{\omega}(x)$ with $n \geq n^{\prime}(\omega, x)$. Then one obtains the first conclusion with $n_{0}^{\prime \prime}(\omega, x)$ being defined by

$$
n_{0}^{\prime \prime}(\omega, x):=\max \left\{n^{\prime}(\omega, x), 1+\left[\frac{1}{\varepsilon} \log \left(2 K_{0} \delta_{*} \ell(\omega, x)\right)\right]\right\} .
$$

By Lemma 8.5, we clearly have

$$
\varlimsup_{n \rightarrow+\infty}-\frac{1}{n} \log \mu_{(\omega, x)}^{\eta^{i} \vee \widetilde{\alpha}_{b(n)}^{0}}\left(\left[\beta_{0}^{n}\right]_{\omega}(x)\right) \leq h_{i} .
$$

On the other hand, by Lemma 8.4 and Proposition 8.6 we have for each $p \in(0,1)$

$$
\begin{aligned}
& \lim _{n \rightarrow+\infty}-\frac{1}{n} \log \mu_{(\omega, x)}^{\eta^{i} \vee \widetilde{\alpha}_{b(n)}^{0}}\left(\left[\beta_{0}^{n}\right]_{\omega}(x)\right)=\varliminf_{n \rightarrow+\infty}-\frac{1}{n} \log \mu_{(\omega, x)}^{\eta^{i} \vee \widetilde{\alpha}_{b(n)}^{0}}\left(\left[\widetilde{\alpha}_{0}^{n+m}\right]_{\omega}(x)\right) \\
= & \lim _{k \rightarrow+\infty} \frac{\lim }{n \rightarrow+\infty}-\frac{1}{n+(k-1) m} \log \mu_{(\omega, x)}^{\eta^{i} \vee \widetilde{\alpha}_{b(n+(k-1) m)}^{0}}\left(\left[\widetilde{\alpha}_{0}^{n+k m}\right]_{\omega}(x)\right) \\
\geq & \lim _{k \rightarrow+\infty} \underline{n \rightarrow+\infty}-\frac{1}{n} \log \mu_{(\omega, x)}^{\eta^{i} \vee \widetilde{\alpha}_{b(n+(k-1) m)}^{0}}\left(B^{i}\left(\omega, x ;[n p], e^{-k m\left(\lambda_{i}-3 \varepsilon_{*}\right)}\right)\right) \\
= & p \cdot \lim _{k \rightarrow+\infty} \underline{h}_{i}^{\prime}\left(\omega, x ; e^{-k m\left(\lambda_{i}-3 \varepsilon_{*}\right)}\right)=p \cdot h_{i} .
\end{aligned}
$$

Letting $p \rightarrow 1$, we obtain the second conclusion.

8.3. Coincidence of pseudo and usual pointwise dimensions. Based on Proposition 8.6, we will prove inductively that for $\mu$-a.e. $(\omega, x)$

$$
\underline{\delta}_{i}^{\prime}(\omega, x)=\bar{\delta}_{i}^{\prime}(\omega, x)=\delta_{i}, \quad i=1,2, \cdots, u,
$$

following the same line of [13, Sect. 5], where the constant $\delta_{i}$ for each $i$ is the pointwise dimension of $\mu$ on $W^{i}$-manifolds defined in [13].

Let $\gamma_{i}=\delta_{i}-\delta_{i-1}$ be the transverse dimension of $\mu$ on $W^{i} / W^{i-1}$ introduced in [13. Sect. 2] for $i=1,2, \cdots, u$ with the convention $\delta_{0}:=0$. First we have

Proposition 8.8. $\underline{\delta}_{1}^{\prime}(\omega, x)=\bar{\delta}_{1}^{\prime}(\omega, x)=\delta_{1}=\gamma_{1}$ for $\mu$-a.e. $(\omega, x)$. 
Proof. We have already known that

$$
\underline{\delta}_{1}^{\prime}(\omega, x) \geq \delta_{1}=\gamma_{1} .
$$

For $\beta=\widetilde{\alpha}_{0}^{m}$ with $m \geq 1$, by Lemmas 8.7 and 8.5 it is clear that

$$
h_{1}=h_{1}^{\prime} \geq \lambda_{1} \bar{\delta}_{1}^{\prime}
$$

i.e., $\bar{\delta}_{1}^{\prime} \leq h_{1} / \lambda_{1}=\delta_{1}$.

Based on Proposition 8.8 we will then prove the coincidence of $\underline{\delta}_{i}^{\prime}$ and $\bar{\delta}_{i}^{\prime}$ with $\delta_{i}$ for $i=2, \cdots, u$ by induction. Let $2 \leq i \leq u$ and assume that we have already proved the coincidence of $\underline{\delta}_{i-1}^{\prime}$ and $\bar{\delta}_{i-1}^{\prime}$ with $\delta_{i-1}$, i.e., for $\mu$-a.e. $(\omega, x)$

$$
\underline{\delta}_{i-1}^{\prime}(\omega, x)=\bar{\delta}_{i-1}^{\prime}(\omega, x)=\delta_{i-1} .
$$

In view of the facts

$$
\underline{\delta}_{i}^{\prime}(\omega, x) \geq \delta_{i}
$$

and

$$
\gamma_{i}=\delta_{i}-\delta_{i-1}=\left(h_{i}-h_{i-1}\right) / \lambda_{i},
$$

the coincidence of $\underline{\delta}_{i}^{\prime}(\omega, x)$ and $\bar{\delta}_{i}^{\prime}(\omega, x)$ with $\delta_{i}$ can be deduced directly from the following proposition.

Proposition 8.9. $\bar{\delta}_{i}^{\prime}-\bar{\delta}_{i-1}^{\prime} \leq\left(h_{i}-h_{i-1}\right) / \lambda_{i}$ for $\mu$-a.e. $(\omega, x)$.

In order to prove Proposition 8.9, we present some basic estimates in the following lemma, which follows readily from the last subsection and Lemma 8.7, and hence whose proof is omitted here.

Lemma 8.10. Let $\varepsilon \in\left(0, \varepsilon_{*}\right)$ be given. Let $\beta=\widetilde{\alpha}_{0}^{m}$ for some integer $m \geq 1$. There exists a Borel function $\widehat{n}: \Omega \times M \rightarrow \mathbf{Z}^{+}$such that the following hold for $\mu$-a.e. $(\omega, x)$ :

(1) $\left[\eta^{i} \vee \beta_{0}^{n}\right]_{\omega}(x) \subset B^{i}\left(\omega, x ; e^{-n\left(\lambda_{i}-9 \varepsilon\right)}\right)$ for any $n \geq \widehat{n}(\omega, x)$;

(2) $-\frac{1}{n} \log \mu_{(\omega, x)}^{\eta^{i-1} \vee \widetilde{\alpha}_{b(n)}^{0}}\left(\left[\beta_{0}^{n}\right]_{\omega}(x)\right) \geq h_{i-1}-\varepsilon \quad$ for any $n \geq \widehat{n}(\omega, x)$;

(3) $-\frac{1}{n} \log \mu_{(\omega, x)}^{\eta^{i} \vee \widetilde{\alpha}_{b(n)}^{0}}\left(\left[\beta_{0}^{n}\right]_{\omega}(x)\right) \leq h_{i}+\varepsilon$ for any $n \geq \widehat{n}(\omega, x)$;

(4) $L:=B^{i-1}\left(\omega, x ; e^{-n\left(\lambda_{i}-9 \varepsilon\right)}\right) \subset \eta_{\omega}^{i-1}(x)$ for any $n \geq \widehat{n}(\omega, x)$;

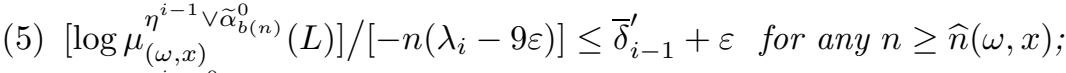

(6) $\left[\log \mu_{(\omega, x)}^{\eta^{i} \vee \widetilde{\alpha}_{b(n)}^{0}}\left(B^{i}\left(\omega, x ; 2 e^{-n\left(\lambda_{i}-9 \varepsilon\right)}\right)\right)\right] /\left[-n\left(\lambda_{i}-9 \varepsilon\right)\right] \geq \bar{\delta}_{i}^{\prime}-\varepsilon \quad$ for infinitely many $n \geq \widehat{n}(\omega, x)$.

Proof of Proposition 8.9. The proof follows the main line of that of [13, Prop. 5.3] with some slight modifications. We present it here for completeness.

Let

$$
\Lambda^{n}:=\{(\omega, x): \widehat{n}(\omega, x) \leq n \text { and }(\omega, x) \text { satisfies }
$$

the requirements (1)-(6) of Lemma 8.10\}.

Clearly $\mu\left(\Lambda^{n}\right) \uparrow 1$ as $n \rightarrow+\infty$. Hence for any $\varepsilon^{\prime} \in(0,1)$ there is an integer $N_{1}$ such that $\Lambda^{\prime}:=\Lambda^{N_{1}}$ has $\mu$-measure not less than $1-\varepsilon^{\prime} / 2$. Then we can assume that, for 
$\mu$-a.e. $(\omega, x), \underline{\delta}_{i-1}^{\prime}(\omega, x ; \bar{\alpha})=\bar{\delta}_{i-1}^{\prime}(\omega, x ; \bar{\alpha})=\delta_{i-1}$ where $\bar{\alpha}:=\widetilde{\alpha} \vee\left\{\Lambda^{\prime}, \Omega \times M \backslash \Lambda^{\prime}\right\}$, i.e.,

$$
\lim _{\rho \rightarrow 0} \frac{\log \mu_{(\omega, x)}^{\eta^{i-1} \vee \bar{\alpha}_{n(\rho)}^{0}}\left(B^{i-1}(\omega, x ; \rho)\right)}{\log \rho}=\bar{\delta}_{i-1}^{\prime}=\delta_{i-1},
$$

where $\bar{\delta}_{i-1}^{\prime}=\delta_{i-1}$ is constant and $n(\rho)$ is any a well-rated function $n:(0,1) \rightarrow \mathbf{Z}^{+}$. Hence for any well-rated function $b: \mathbf{Z}^{+} \rightarrow \mathbf{Z}^{+}$one has

$$
\lim _{n \rightarrow+\infty}-\frac{1}{n} \log \mu_{(\omega, x)}^{\eta^{i-1} \vee \widetilde{\alpha}_{b(n)}^{0}}\left(B^{i-1}\left(\omega, x ; e^{-n\left(\lambda_{i}-9 \varepsilon\right)}\right) \cap \Lambda_{\omega}^{\prime}\right)=\left(\lambda_{i}-9 \varepsilon\right) \cdot \bar{\delta}_{i-1}^{\prime}
$$

for $\mu$-a.e. $(\omega, x) \in \Lambda^{\prime}$. There is thus another integer $N_{2} \geq N_{1}$ and a subset $\bar{\Lambda} \subset \Lambda^{\prime}$ of $\mu$-measure not less than $1-\varepsilon^{\prime}$ such that, for any $(\omega, x) \in \bar{\Lambda}$ and any $n \geq N_{2}$,

$$
\mu_{(\omega, x)}^{\eta^{i-1} \vee \widetilde{\alpha}_{b(n)}^{0}}\left(L \cap \Lambda_{\omega}^{\prime}\right) \geq e^{-n\left(\lambda_{i}-9 \varepsilon\right)\left(\bar{\delta}_{i-1}^{\prime}+\varepsilon\right)}
$$

with $L=B^{i-1}\left(\omega, x ; e^{-n\left(\lambda_{i}-9 \varepsilon\right)}\right)$. Now we arbitrarily fix a point $(\omega, x) \in \bar{\Lambda}$. By Lemma 8.10 one has

$$
\mu_{(\omega, x)}^{\eta^{i-1} \vee \widetilde{\alpha}_{b(n)}^{0}}\left(\left[\beta_{0}^{n}\right]_{\omega}(y)\right)=\mu_{(\omega, y)}^{\eta^{i-1} \vee \widetilde{\alpha}_{b(n)}^{0}}\left(\left[\beta_{0}^{n}\right]_{\omega}(y)\right) \leq e^{-n\left(h_{i-1}-\varepsilon\right)}
$$

for any $y \in L \cap \Lambda_{\omega}^{\prime} \cap\left[\widetilde{\alpha}_{b(n)}^{0}\right]_{\omega}(x) \subset\left[\eta^{i-1} \vee \widetilde{\alpha}_{b(n)}^{0}\right]_{\omega}(x)$ and $n \geq N_{2}$. Hence, by Lemma $8.10(5)$,

$$
\begin{aligned}
& \#\left\{\left[\beta_{0}^{n}\right]_{\omega}(y): y \in L \cap \Lambda_{\omega}^{\prime} \cap\left[\widetilde{\alpha}_{b(n)}^{0}\right]_{\omega}(x)\right\} \\
\geq & \mu_{(\omega, x)}^{\eta^{i-1} \vee \widetilde{\alpha}_{b(n)}^{0}}\left(L \cap \Lambda_{\omega}^{\prime}\right) / e^{-n\left(h_{i-1}-\varepsilon\right)} \\
\geq & \exp \left\{n\left[h_{i-1}-\varepsilon-\left(\lambda_{i}-9 \varepsilon\right)\left(\bar{\delta}_{i-1}^{\prime}+\varepsilon\right)\right]\right\} .
\end{aligned}
$$

On the other hand, according to Lemma 8.10(1), we have

$$
\left[\eta^{i} \vee \beta_{0}^{n}\right]_{\omega}(y) \subset B^{i}\left(\omega, y ; e^{-n\left(\lambda_{i}-9 \varepsilon\right)}\right), \quad \forall n \geq N_{2},
$$

for any $(\omega, y) \in \Lambda^{\prime}$ and hence also for any $y \in L \cap \Lambda_{\omega}^{\prime}$. Clearly $d_{(\omega, x)}^{i}(x, y) \leq$ $d_{(\omega, x)}^{i-1}(x, y)$ for any $y \in L$. Therefore

$$
\left[\eta^{i} \vee \beta_{0}^{n}\right]_{\omega}(y) \subset B^{i}\left(\omega, x ; 2 e^{-n\left(\lambda_{i}-9 \varepsilon\right)}\right), \quad \forall y \in L \cap \Lambda_{\omega}^{\prime} \text { and } n \geq N_{2} .
$$

Thus, noting that $L \subset \eta_{\omega}^{i-1}(x) \subset \eta_{\omega}^{i}(x)$, we have

$$
\begin{array}{ll} 
& \log \mu_{(\omega, x)}^{\eta^{i} \vee \widetilde{\alpha}_{b(n)}^{0}}\left(B^{i}\left(\omega, x ; 2 e^{-n\left(\lambda_{i}-9 \varepsilon\right)}\right)\right) \\
\geq \quad & \log \#\left\{\left[\eta^{i} \vee \beta_{0}^{n}\right]_{\omega}(y): y \in L \cap \Lambda_{\omega}^{\prime} \cap\left[\widetilde{\alpha}_{b(n)}^{0}\right]_{\omega}(x)\right\} \\
& +\log \min _{y} \mu_{(\omega, x)}^{\eta^{i} \vee \widetilde{\alpha}_{b(n)}^{0}}\left(\left[\eta^{i} \vee \beta_{0}^{n}\right]_{\omega}(y)\right) \\
\geq \quad & \log \#\left\{\left[\beta_{0}^{n}\right]_{\omega}(y): y \in L \cap \Lambda_{\omega}^{\prime} \cap\left[\widetilde{\alpha}_{b(n)}^{0}\right]_{\omega}(x)\right\} \\
& +\log \min _{y} \mu_{(\omega, x)}^{\eta^{i} \vee \widetilde{\alpha}_{b(n)}^{0}}\left(\left[\beta_{0}^{n}\right]_{\omega}(y)\right) \\
\geq & -n\left[h_{i}-h_{i-1}+2 \varepsilon+\left(\lambda_{i}-9 \varepsilon\right)\left(\bar{\delta}_{i-1}^{\prime}+\varepsilon\right)\right] .
\end{array}
$$

Then by Lemma $8.10(6)$ we obtain for any $(\omega, x) \in \bar{\Lambda}$

$$
\left(\lambda_{i}-9 \varepsilon\right)\left(\bar{\delta}_{i}^{\prime}-\bar{\delta}_{i-1}^{\prime}-2 \varepsilon\right) \leq h_{i}-h_{i-1}+2 \varepsilon,
$$

which implies Proposition 8.9 by letting $\varepsilon \rightarrow 0$ and then $\varepsilon^{\prime} \rightarrow 0$. 


\section{REFERENCES}

[1] L. Arnold. Random Dynamical Systems. Springer, 1998. MR.1723992 (2000m:37087)

[2] J. Bahnmüller and P.-D. Liu. Characterization of measures satisfying Pesin's entropy formula for random dynamical systems. J. Dynam. Diff. Equ. 10(3)(1998), 425-448. MR1646606 (99j:58118)

[3] L. Barreira, Y. Pesin and J. Schmeling. Dimension and product structure of hyperbolic measures. Ann. Math. 149 (1999), 755-783. MR1709302 (2000f:37027)

[4] T. Bogenschütz. Entropy, pressure, and a variational principle for random dynamical systems. Random and Computational Dynamics 1 (1992), 219-227. MR1181382 (93k:28023)

[5] T. Bogenschütz. Equilibrium states for random dynamical systems. Ph.D. thesis, Institut für Dynamische Systeme, Universität Bremen (1993).

[6] M. de Guzmán. Differentiation of Integrals in $\mathbf{R}^{n}$. Lecture Notes in Math. 481, SpringerVerlag, New York, 1975. MR0457661 (56:15866)

[7] Y. Kifer. Ergodic Theory of Random Transformations. Birkhäuser, Boston, 1986. MR0884892 (89c:58069)

[8] F. Ledrappier and L.-S. Young. The metric entropy of diffeomorphisms. Part II: Relations between entropy, exponents and dimension. Ann. Math. 122 (1985), 540-574. MR0819557 (87i:58101b)

[9] P.-D. Liu. (Survey) Dynamics of random transformations: smooth ergodic theory. Ergod. Th. Dynam. Sys. 21 (2001), 1279-1319. MR 1855833(2002g:37024)

[10] P.-D. Liu. Random perturbations of Axiom A basic sets. J. Stat. Phys. 90(1/2) (1998), 467490. MR1611096 (99a:58108)

[11] P.-D. Liu and M. Qian. Smooth Ergodic Theory of Random Dynamical Systems. Lect. Not. Math. 1606, Springer-Verlag, 1995. MR1369243 (96m:58139)

[12] Ya. B. Pesin, Dimension Theory in Dynamical Systems: Contemporary Views and Applications, Chicago Lectures in Mathematics Series, the University of Chicago Press, Chicago and London, 1997. MR.1489237 (99b:58003)

[13] M. Qian, M.-P. Qian and J.-S. Xie. Entropy formula for random dynamical systems: Relations between entropy, exponents and dimension. Ergod. Th. Dynam. Sys. 23 (2003), no. 6, 19071931. MR2032494(2004m:37096)

[14] V. A. Rokhlin. On the foundamental ideas of measure theory. Amer. Math. Soc. Transl. (1) 10 (1962), 1-52.

[15] V. A. Rokhlin. Lectures on the entropy theory of measure-preserving transformations. Russ. Math. Surveys 22:5 (1967), 1-54.

School of Mathematical Sciences, Peking University, 100871 Beijing, People's RePUBLIC OF CHINA

E-mail address: lpd@pku.edu.cn

School of Mathematical Sciences, Peking University, 100871 Beijing, People's RePUBlic OF CHINA

E-mail address: js_xie@yahoo.com.cn

Current address: Institute of Mathematics, Academy of Mathematics and Systems Science, Chinese Academy of Sciences, 100080 Beijing, People's Republic of China 